\title{
A Caporaso-Harris type formula for Welschinger invariants of real toric Del Pezzo surfaces
}

\author{
Ilia Itenberg, Viatcheslav Kharlamov and Eugenii Shustin*
}

\begin{abstract}
We define a series of relative tropical Welschinger-type invariants of real toric surfaces. In the Del Pezzo case, these invariants can be seen as real tropical analogs of relative GromovWitten invariants, and are subject to a recursive formula. As application we obtain new formulas for Welschinger invariants of real toric Del Pezzo surfaces.
\end{abstract}

Mathematics Subject Classification (2000). Primary 14N10; Secondary 14P05, 14N35, $51 \mathrm{~N} 35$.

Keywords. Tropical curves, enumerative geometry, Welschinger invariants, Caporaso-Harris formula, toric surfaces.

\section{Introduction}

Welschinger invariants of real rational symplectic four-manifolds [10], [11] represent one of the most interesting and intriguing objects in real enumerative geometry. In the case of a real unnodal (i.e., not containing any rational $(-n)$-curve, $n \geq 2$ ) Del Pezzo surface $\Sigma$ the Welschinger invariants count, with appropriate weights \pm 1 , the real rational curves which belong to an ample linear system $|D|$ and pass through a given generic conjugation-invariant set of $c_{1}(\Sigma) \cdot D-1$ points in $\Sigma$. In this paper we consider only the invariants corresponding to sets of real points.

Our goal is to provide recursive formulas which calculate the Welschinger invariants of toric Del Pezzo surfaces equipped with the tautological real structure. The formulas we obtain are similar to those proved by L. Caporaso and J. Harris [1] for relative Gromov-Witten invariants of $\mathbb{P}^{2}$.

\footnotetext{
${ }^{*}$ The authors were partially supported by a grant from the Ministry of Science and Technology, Israel, and Ministère des Affaires Etrangères, France. The first two authors were partially funded by the ANR-05-005301 grant of Agence Nationale de la Recherche and a grant of Université Louis Pasteur, Strasbourg. The first and the third author enjoyed a support from the Hermann-Minkowski-Minerva Center for Geometry at the Tel Aviv University. The third author acknowledges a support from the grant no. 465/04 from the Israel Science Foundation.
} 
We use the technique of tropical geometry and follow ideas of A. Gathmann and H. Markwig [2], [3] who suggested a tropical version of the Caporaso-Harris formula and its tropical proof. We suitably adapt the tropical count to the real setting and introduce tropical, multi-component and irreducible, Welschinger numbers for relative constraints and arbitrary genus. We check their invariance (Theorem 1 in Section 3.1) and prove that these tropical invariants satisfy Caporaso-Harris type formulas (Theorem 3 for the multi-component invariants and Theorem 4 for irreducible invariants, Section 3; a reformulation with generating functions is presented in Section 6.1). In the case when the set of relative constraints is empty these invariants coincide with the genuine Welschinger invariants. As a by-product, we establish some monotonicity of the Welschinger invariants and give a new proof of their positivity (Corollaries 4 and 5, Section 3.3).

The paper is organized as follows. In Section 2 we remind definitions and basic facts concerning Welschinger invariants and plane tropical curves. Section 3 contains the definition of tropical relative Welschinger numbers, the statements of the main results and few corollaries. We prove the invariance of tropical relative Welschinger numbers in Section 4 and the recursive formulas in Section 5. Section 6 is devoted to concluding remarks. Our main results are stated in terms of embedded plane tropical curves, while the proofs go essentially through the parameterized incarnation of these curves, and starting from Section 4 we put a special attention to be maximally possible consistent with existing in the literature notions and statements concerning these two different categories. In particular, a part of Section 4 is devoted to various types of genericity conditions and their comparison.

To conclude this short introduction, we would like to emphasize a certain, challenging in our opinion, difference between the real and complex cases. Namely, Gathmann-Markwig's count of tropical curves in the tropical version of CaporasoHarris formula is in a strict correspondence (in the sense of modified Mikhalkin's correspondence theorem [7], see [2], [3]) with the count of complex algebraic curves in the original Caporaso-Harris formula. In particular, the invariance of the terms in Caporaso-Harris formula explains (and implies) the invariance of the terms in the tropical version of Caporaso-Harris formula proposed by Gathmann and Markwig. On the contrary, we do not know how to lift up invariantly the terms entering the formulas suggested in the present paper (except those which lead to the genuine Welschinger invariants). One of the difficulties is that such a lift, if it exists, can not be formulated in purely topological terms, see Section 6 .

Acknowledgements. A considerable part of this work was done during our visits to the Max-Planck-Institut für Mathematik, Bonn. We thank MPIM for the hospitality and excellent working conditions. 


\section{Preliminaries}

2.1. Welschinger invariants of Del Pezzo surfaces. We remind here the definition of Welschinger invariants [10], [11] restricting ourselves to a particular situation. Let $\Sigma$ be a real unnodal (i.e., not containing any rational $(-n)$-curve, $n \geq 2$ ) Del Pezzo surface with a connected real part $\mathbb{R} \Sigma$, and let $D \subset \Sigma$ be a real ample divisor. Consider a generic set $\omega$ of $c_{1}(\Sigma) \cdot D-1$ real points of $\Sigma$. The set $R(D, \omega)$ of real $C \in|D|$ passing through the points of $\omega$ is finite, and all these curves are nodal and irreducible. (In fact, the listed properties of $R(D, \omega)$ can be taken here as a definition of the term 'generic'.) Due to the Welschinger theorem [10], [11] (and the genericity of the complex structure on $\Sigma$ ), the number

$$
\mathrm{W}(\Sigma, D, \omega)=\sum_{C \in R(D, \omega)}(-1)^{s(C)},
$$

where $s(C)$ is the number of solitary nodes of $C$ (i.e., real points, where a local equation of the curve can be written over $\mathbb{R}$ in the form $x^{2}+y^{2}=0$ ), does not depend on the choice of a generic set $\omega$. We denote this Welschinger invariant by $\mathrm{W}(\Sigma, D)$.

2.2. Divisors on toric Del Pezzo surfaces. There are five toric unnodal Del Pezzo surfaces: the projective plane $\mathbb{P}^{2}$, the product $\mathbb{P}^{1} \times \mathbb{P}^{1}$ of projective lines, and $\mathbb{P}^{2}$ with $k$ blown up generic points, $k=1$, 2, or 3 ; the latter three surfaces are denoted by $\mathbb{P}_{k}^{2}$. Let $E_{1}, \ldots, E_{k}$ be the exceptional divisors of $\mathbb{P}_{k}^{2} \rightarrow \mathbb{P}^{2}$ and $L \subset \mathbb{P}_{k}^{2}$ the pull back of a generic straight line.

Let $\Sigma$ be one of these surfaces. An ample divisor on $\Sigma$ defines a linear system, which in suitable toric coordinates is generated by monomials $x^{i} y^{j}$, where $(i, j)$ ranges over all the integer points of a polygon $\Delta(D)$ of the following form. If $\Sigma=\mathbb{P}^{2}$ and $D=d \mathbb{P}^{1}$, then $\Delta(D)$ is the triangle with vertices $(0,0),(d, 0)$, and $(0, d)$. If $\Sigma=\mathbb{P}^{1} \times \mathbb{P}^{1}$ and $D$ is of bi-degree $\left(d_{1}, d_{2}\right)$, then $\Delta(D)$ is the rectangle with vertices $(0,0),\left(d_{1}, 0\right),\left(d_{1}, d_{2}\right)$, and $\left(0, d_{2}\right)$. If $\Sigma=\mathbb{P}_{k}^{2}, k=1,2$, or 3 , and $D=d L-\sum_{i=1}^{k} d_{i} E_{i}$, then $\Delta(D)$ is respectively the trapeze with vertices $(0,0),\left(d-d_{1}, 0\right),\left(d-d_{1}, d_{1}\right),(0, d)$, or the pentagon with vertices $\left(d_{2}, 0\right)$, $\left(d-d_{1}, 0\right),\left(d-d_{1}, d_{1}\right),(0, d),\left(0, d_{2}\right)$, or the hexagon with vertices $\left(d_{2}, 0\right)$, $\left(d-d_{1}, 0\right),\left(d-d_{1}, d_{1}\right),\left(d_{3}, d-d_{3}\right),\left(0, d-d_{3}\right),\left(0, d_{2}\right)$ (see Figure 1$)$. The slopes of the sides of $\Delta(D)$ are $0,-1$, or $\infty$.

2.3. Plane tropical curves. In Sections 2.3 and 2.4 we remind definitions and basic facts concerning plane tropical curves (cf. [7], [3]), and fix the notation.

Let $\Delta \subset \mathbb{R}^{2}$ be a nondegenerate convex lattice polygon, i.e., a convex polygon with integer vertices and non-empty interior. A convex piecewise-linear function

$$
F: \mathbb{R}^{2} \rightarrow \mathbb{R}, \quad F(x)=\max _{\iota \in \Delta \cap \mathbb{Z}^{2}}\left(\langle\iota, x\rangle+c_{\iota}\right), \quad \text { where } c_{\iota} \in \mathbb{R},
$$



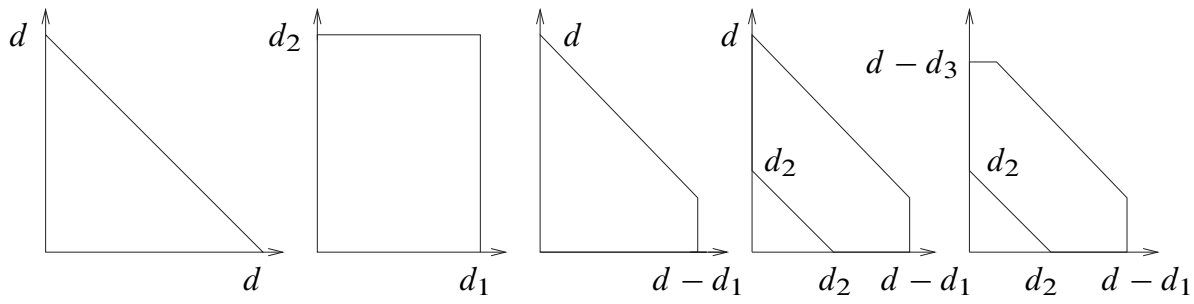

Figure 1. Polygons associated with Del Pezzo surfaces.

is called a tropical polynomial with Newton polygon $\Delta$. Consider the corner locus $A_{F} \subset \mathbb{R}^{2}$ of $F$ (i.e., the subset of $\mathbb{R}^{2}$ where $F$ is not smooth). The set $A_{F}$ is naturally stratified and defines a subdivision $\Theta_{F}$ of $\mathbb{R}^{2}$. The 0 - and 1-dimensional elements of the stratification of $A_{F}$ are called, respectively, its vertices and edges.

The Legendre transform takes $F$ to a convex piecewise-linear function $\varrho_{F}: \Delta \rightarrow$ $\mathbb{R}$, whose linearity domains $\Delta_{1}, \ldots, \Delta_{N}$ are convex lattice subpolygons of $\Delta$, and whose graph is the lower part of the polytope $\operatorname{conv}\left\{\left(l,-c_{l}\right) \in \mathbb{R}^{3}, \iota \in \Delta \cap \mathbb{Z}^{2}\right\}$. The polygons $\Delta_{1}, \ldots, \Delta_{N}$ give rise to a subdivision $S_{F}$ of $\Delta$. This subdivision is dual to $\Theta_{F}$ in the following sense: there is a one-to-one correspondence $\mathscr{D}$ between the elements of $S_{F}$ and the elements of $\Theta_{F}$ such that

- $D$ sends any vertex of $S_{F}$ to a 2-cell of $\Theta_{F}$, any edge of $S_{F}$ to an edge of $\Theta_{F}$, and any polygon of $S_{F}$ to a vertex of $\Theta_{F}$;

- for any edge $e$ of $S_{F}$, the edge $\mathscr{D}(e)$ is orthogonal to $e$;

- $D$ reverses the incidence relation.

Each edge $e$ of $A_{F}$ can be equipped with a weight $w(e)$ equal to the lattice length (i.e., the number of integer points diminished by 1) of the dual edge in $S_{F}$. The stratified set $A_{F}$ whose edges are equipped with the corresponding weights is called the tropical curve associated with the tropical polynomial $F$. One says that $A_{F}$ is a plane tropical curve with Newton polygon $\Delta$. A plane tropical curve determines its Newton polygon $\Delta$ and the dual subdivision of $\Delta$ uniquely up to translation.

Notice that the unbounded edges of $A_{F}$ are dual to the edges of $S_{F}$ lying on the boundary $\partial \Delta$ of $\Delta$. The unbounded edges of $A_{F}$ are called ends of $A_{F}$. The unbounded edges of $A_{F}$ dual to the edges of $S_{F}$ which are contained in a side $\sigma$ of $\Delta$ are called $\sigma$-ends.

Any edge of a plane tropical curve $A_{F}$ has rational slope, and for any vertex $v$ of $A_{F}$ one has the balancing condition

$$
w\left(e_{1}\right) u\left(v, e_{1}\right)+\cdots+w\left(e_{k}\right) u\left(v, e_{k}\right)=0,
$$

where $e_{1}, \ldots, e_{k}$ are the edges adjacent to $v$, and $u\left(v, e_{i}\right)$ is the primitive integer vector starting at $v$ and directed along $e_{i}$. 
The sum $A_{F^{(1)}}+\cdots+A_{F^{(n)}}$ of plane tropical curves $A_{F^{(1)}}, \ldots, A_{F^{(n)}}$ is the plane tropical curve defined by the tropical polynomial $F^{(1)}+\cdots+F^{(n)}$. The underlying set of $A_{F^{(1)}}+\cdots+A_{F^{(n)}}$ is the union of underlying sets of $A_{F^{(1)}}, \ldots, A_{F^{(n)}}$, and the weight of any edge of $A_{F^{(1)}}+\cdots+A_{F^{(n)}}$ is equal to the sum of the weights of the corresponding edges of summands. A tropical curve in $\mathbb{R}^{2}$ is reducible if it is the sum of two proper tropical subcurves. A non-reducible tropical curve in $\mathbb{R}^{2}$ is called irreducible. A tropical curve $A_{F}$ is nodal, if any polygon of the dual subdivision $S_{F}$ is either triangle, or parallelogram. The number of double points of a nodal tropical curve $A_{f}$ with Newton polygon $\Delta$ is the sum of the number of parallelograms in $S_{F}$ and the number of integer points which belong to the interior of $\Delta$ and are not vertices of $S_{F}$.

The multi-set of vectors $\{w(e) u(e)\}$, where $e$ runs over the ends of a plane tropical curve $A_{F}$ and each primitive integer vector $u(e)$ is directed along $e$ to infinity, is called the degree of $A_{F}$.

We extend the plane $\mathbb{R}^{2}$ up to $\widehat{\mathbb{R}}^{2}=\mathbb{T} \times \mathbb{R}$, where $\mathbb{T}=\mathbb{R} \cup\{-\infty\}$ is equipped with the topology making $\mathbb{T}$ homeomorphic to $[0,+\infty)$ via a logarithmic map, and correspondingly extend any tropical curve in $\mathbb{R}^{2}$ by attaching a vertex on $L_{-\infty}=$ $\{-\infty\} \times \mathbb{R}$ to any horizontal negatively directed end of the curve.

2.4. Parameterizations of plane tropical curves. Let $\bar{\Gamma}$ be a finite connected graph without divalent vertices, and $\mathcal{V}$ a collection of certain univalent vertices of $\bar{\Gamma}$. Put $\Gamma=\bar{\Gamma} \backslash \mathcal{V}$. Denote by $\Gamma_{0}^{0}$ the set of non-univalent vertices of $\Gamma$, by $\Gamma^{1}$ the set of edges of $\Gamma$, and by $\Gamma_{\infty}^{1}$ the set of edges of $\Gamma$ such that the corresponding edges of $\bar{\Gamma}$ terminate at univalent vertices (we call such edges of $\Gamma$ the ends).

A parameterized plane tropical curve is a triple $(\Gamma, w, h)$, where $w: \Gamma^{1} \rightarrow \mathbb{N}$ is a function (called weight function) and $h: \Gamma \rightarrow \widehat{\mathbb{R}}^{2}$ is a continuous proper map such that

- for any $E \in \Gamma^{1}$, the restriction of $h$ to $E$ is an embedding into a straight line with a rational slope,

- if $E \in \Gamma_{\infty}^{1}$ and $E$ terminates at a univalent vertex $V \in \Gamma$, then $h(E)$ lies in a horizontal line and $h(V) \in L_{-\infty}$,

- for any $V \in \Gamma_{0}^{0}$, the union

$$
\bigcup_{\substack{E \in \Gamma^{1} \\ V \in \partial E}} h(E)
$$

is not contained in a line, and one has the balancing condition

$$
\sum_{\substack{E \in \Gamma^{1} \\ V \in \partial E}} w(E) u(V, E)=0,
$$


where $u(V, E)$ is the primitive integer vector starting at $h(V)$ and directed along $h(E)$.

The ends of $\Gamma$ which are adjacent to univalent vertices of $\Gamma$ are called left. Due to the properness of $h$, the non-left ends of $\Gamma$ are mapped onto half-lines which are not horizontal negatively directed.

The multi-set of vectors $\left\{w(E) u(E): E \in \Gamma_{\infty}^{1}\right\}$, where each primitive integer vector $u(E)$ is directed along $h(E)$ to infinity, is called the degree of $(\Gamma, w, h)$.

A parameterized plane tropical curve $(\Gamma, w, h)$ is a parameterization of a plane tropical curve $T$ if

- the image under $h$ of any vertex of $\Gamma$ is a vertex of $T$,

- the closure of the image under $h$ of any edge of $\Gamma$ is the closure of a union of edges of $T$,

- the weight of any edge $e$ of $T$ is equal to $w\left(E_{1}\right)+\cdots+w\left(E_{n}\right)$, where $E_{1}, \ldots, E_{n}$ are the edges of $\Gamma$ whose images under $h$ contain $e$.

Any parameterized plane tropical curve is a parameterization of a unique plane tropical curve. Notice that if $(\Gamma, w, h)$ is a parameterization of a plane tropical curve $T$, the curve $T$ might have vertices that are not images under $h$ of vertices of $\Gamma$.

The genus of a parameterized plane tropical curve $(\Gamma, w, h)$ is the first Betti number $b_{1}(\Gamma)$ of $\Gamma$. If a plane tropical curve $T$ is irreducible, the minimal genus of its parameterizations is called the genus of $T$ and is denoted by $g(T)$.

The degree of an irreducible nodal plane tropical curve $T$ coincides with the degree of any parameterization of $T$. If $T$ is an irreducible nodal plane tropical curve, then any minimal genus parameterization $(\Gamma, w, h)$ of $T$ is simple, that is, any vertex of $\Gamma$ has valency either 3 , or 1 . For any two simple parameterizations $(\Gamma, w, h)$ and $\left(\Gamma^{\prime}, w^{\prime}, h^{\prime}\right)$ of a given irreducible nodal plane tropical curve, there exists a homeomorphism $\varphi: \Gamma \rightarrow \Gamma^{\prime}$ such that $h=h^{\prime} \circ \varphi$ and $w(E)=w^{\prime}(\varphi(E))$ for any $E \in \Gamma^{1}$.

If $T$ is a nodal plane tropical curve, then each edge of $T$ is contained in a unique irreducible subcurve of $T$. In particular, a nodal plane tropical curve is uniquely represented as a sum of its irreducible subcurves. (Notice that this statement is not true without the nodality assumption.) Furthermore, any irreducible subcurve of a nodal plane tropical curve is nodal. The genus $g(T)$ of a nodal plane tropical curve $T$ is $g\left(T^{(1)}\right)+\cdots+g\left(T^{(n)}\right)-n+1$, where $T^{(1)}, \ldots, T^{(n)}$ are all the irreducible subcurves of $T$. If $T$ is a nodal plane tropical curve with Newton polygon $\Delta$, then the genus of $T$ is equal to the difference between the number of vertices of the dual subdivision $S_{T}$ which belong to the interior of $\Delta$ and the number of parallelograms in $S_{T}$. In particular, the sum of the genus of $T$ and the number of double points of $T$ is equal to the number of interior integer points of $\Delta$. 


\section{Tropical Welschinger invariants}

3.1. Tropical Welschinger invariants of toric surfaces. Denote by $\ell$ the semigroup of sequences $\alpha=\left(\alpha_{1}, \alpha_{2}, \ldots\right) \in \mathbb{Z}^{\infty}$ with nonnegative terms and finite $l_{1}$-norm $\|\alpha\|=\sum_{i} \alpha_{i}$. Each element of $\ell$ contains only finitely many non-zero terms, so in the description of concrete sequences we omit zero terms after the last non-zero one. The only exception concerns the zero element of $\mathcal{C}$ (the sequence with all the terms equal to zero). This element is denoted by (0). For an element $\alpha$ in $\ell$, put $J \alpha=\sum_{i=1}^{\infty}(2 i-1) \alpha_{i}$. Define in $\zeta$ the following natural partial order: if each term of a sequence $\alpha$ is greater than or equal to the corresponding term of a sequence $\beta$, then we say that $\alpha$ is greater than or equal to $\beta$ and write $\alpha \geq \beta$. For two elements $\alpha=\left(\alpha_{1}, \alpha_{2}, \ldots\right)$ and $\beta=\left(\beta_{1}, \beta_{2}, \ldots\right)$ of $\zeta$ such that $\alpha \geq \beta$, the sequence $\alpha-\beta=\left(\alpha_{1}-\beta_{1}, \alpha_{2}-\beta_{2}, \ldots\right)$ is an element of $\mathcal{C}$.

Let $\Delta \subset \mathbb{R}^{2}$ be a nondegenerate convex lattice polygon, and $\sigma$ the intersection of $\Delta$ with its left vertical supporting line. Assume that $\sigma$ is a not a point. In this case, we say that $\Delta$ is left-nondegenerate. Pick two elements $\alpha$ and $\beta$ in $\mathcal{C}$ such that $J \alpha+J \beta=|\sigma|$, where $|\sigma|$ is the lattice length of $\sigma$. Fix an integer $g$, and put

$$
r=|\partial \Delta|-|\sigma|+\|\alpha\|+\|\beta\|+g-1,
$$

where $|\partial \Delta|$ is the lattice length of the boundary of $\Delta$. Assume that $r>\|\alpha\|$.

Consider the space $\Omega(\Delta, \alpha, \beta, g)=\left(L_{-\infty}\right)^{\|\alpha\|} \times\left(\mathbb{R}^{2}\right)^{r-\|\alpha\|}$ formed by the (ordered) configurations $\boldsymbol{p}=\left(\boldsymbol{p}^{\mathrm{b}}, \boldsymbol{p}^{\sharp}\right)$ of $r$ points in $\widehat{\mathbb{R}}^{2}$ such that $\boldsymbol{p}^{\mathrm{b}}=\left(p_{1}, \ldots, p_{\|\alpha\|}\right)$ is a sequence of $\|\alpha\|$ points on $L_{-\infty}$, and $p^{\sharp}=\left(p_{\|\alpha\|+1}, \ldots, p_{r}\right)$ is a sequence of $|\partial \Delta|-|\sigma|+\|\beta\|+g-1$ points in $\mathbb{R}^{2}$. For any $\boldsymbol{p} \in \Omega(\Delta, \alpha, \beta, g)$, introduce the set $\mathcal{T}(\Delta, \alpha, \beta, g, \boldsymbol{p})$ of nodal plane tropical curves $T \subset \widehat{\mathbb{R}}^{2}$ satisfying the following conditions:

- $T$ has $\Delta$ as Newton polygon and is of genus $g$;

- all the $\sigma^{\prime}$-ends of $T$, where $\sigma^{\prime} \neq \sigma$, have weight 1 ;

- the number of $\sigma$-ends of $T$ is equal to $\|\alpha+\beta\|$, and precisely $\alpha_{i}+\beta_{i}$ of them have weight $2 i-1, i \geq 1$;

- any irreducible subcurve of $T$ has a $\sigma$-end;

- $T$ passes through all the points of $\boldsymbol{p}$, and any point $p_{k} \in \boldsymbol{p}^{\mathrm{b}}$ is contained in a $\sigma$-end of weight $2 i_{k}-1$, where the positive integer $i_{k}$ is determined by the inequalities $\sum_{j<i_{k}} \alpha_{j}<k \leq \sum_{j \leq i_{k}} \alpha_{j}$.

The first three conditions completely describe the degree of $T$, and further on we denote this degree by $\Delta^{\alpha, \beta}$. For any $T \in \mathcal{T}(\Delta, \alpha, \beta, g, \boldsymbol{p})$ one has

$$
r=\# \operatorname{End}(T)+g-1,
$$


where \#End $(T)$ is the number of ends of $T$. For a generic $p \in \Omega(\Delta, \alpha, \beta, g)$, the set $\mathcal{T}(\Delta, \alpha, \beta, g, \boldsymbol{p})$ is finite. This assertion can be proved similarly to the corresponding one in [7]. A more precise statement and a proof is found in Section 4.8.

Let $T$ be a curve in $\mathcal{T}(\Delta, \alpha, \beta, g, \boldsymbol{p})$. If $T$ does not have edges of even weight, put $\mathrm{W}(T)=(-1)^{s}$, where $s$ is the total number of integer points lying in the interior of the triangles of the subdivision $S_{T}$ of $\Delta$. Otherwise, put $\mathrm{W}(T)=0$. The number $\mathrm{W}(T)$ is called the Welschinger multiplicity of $T$. Note that for a reducible tropical curve $T \in \mathcal{T}(\Delta, \alpha, \beta, g, \boldsymbol{p})$, its Welschinger multiplicity is the product of the Welschinger multiplicities of all the irreducible subcurves of $T$.

The Welschinger multiplicity can be also defined for any simply parameterized plane tropical curve $(\Gamma, w, h)$. Namely, let $V$ be a vertex in $\Gamma_{0}^{0}$. If the weights of all the edges of $\Gamma$ that are adjacent to $V$ are odd, denote by $s(V)$ the number of interior integer points in the triangle built on the vectors $w\left(E_{1}\right) u\left(V, E_{1}\right)$ and $w\left(E_{2}\right) u\left(V, E_{2}\right)$, where $E_{1}, E_{2}$ is a pair of edges of $\Gamma$ adjacent to $V$, and put $\mathrm{W}(V)=(-1)^{s(V)}$. Otherwise, put $\mathrm{W}(V)=0$. The number $\prod_{V \in \Gamma_{0}^{0}} \mathrm{~W}(V)$ is called the Welschinger multiplicity of $(\Gamma, w, h)$ and is denoted by $\mathrm{W}(\Gamma, w, h)$. If $(\Gamma, w, h)$ is a simple parameterization of a nodal plane tropical curve $T$, then $\mathrm{W}(\Gamma, w, h)=\mathrm{W}(T)$.

Denote by $\mathcal{T}^{\text {irr }}(\Delta, \alpha, \beta, g, \boldsymbol{p})$ the set of irreducible curves in $\mathcal{T}(\Delta, \alpha, \beta, g, \boldsymbol{p})$, and put

$$
\mathrm{W}(\Delta, \alpha, \beta, g, \boldsymbol{p})=\sum_{T \in \mathcal{T}(\Delta, \alpha, \beta, g, \boldsymbol{p})} \mathrm{W}(T),
$$

and

$$
\mathrm{W}^{\mathrm{irr}}(\Delta, \alpha, \beta, g, \boldsymbol{p})=\sum_{T \in \mathcal{T} \text { irr }(\Delta, \alpha, \beta, g, \boldsymbol{p})} \mathrm{W}(T)
$$

Theorem 1. The numbers $\mathrm{W}(\Delta, \alpha, \beta, g, \boldsymbol{p})$ and $\mathrm{W}^{\mathrm{irr}}(\Delta, \alpha, \beta, g, \boldsymbol{p})$ do not depend on the choice of a generic configuration $\boldsymbol{p} \in \Omega(\Delta, \alpha, \beta, g)$.

The word 'generic' in the statement of Theorem 1 means that the configurations are taken in an open dense subset of $\Omega(\Delta, \alpha, \beta, g)$. This subset is explicitly described in Section 4.8 (see the definition of multi-tropically generic configurations).

Due to Theorem 1, one can skip $\boldsymbol{p}$ in the notation of the above numbers. The numbers $\mathrm{W}(\Delta, \alpha, \beta, g)$ (resp., $\left.\mathrm{W}^{\mathrm{irr}}(\Delta, \alpha, \beta, g)\right)$ are called multi-component (resp., irreducible) relative tropical Welschinger invariants.

The following statement is a corollary of Mikhalkin's correspondence theorem [7]. 
Theorem 2 (see [6], Theorem 6, and [8], Proposition 6.1). Let $\Sigma$ be a toric unnodal Del Pezzo surface equipped with its tautological real structure, $D \subset \Sigma$ an ample divisor, and $\Delta$ is a polygon $\operatorname{SL}(2, \mathbb{Z})$-and-translation equivalent to the polygon $\Delta(D)$ defined in Section 2.2. Assume that $\Delta$ is left-nondegenerate. Then

$$
\mathrm{W}^{\mathrm{irr}}(\Delta,(0),(|\sigma|), 0)=\mathrm{W}(\Sigma, D),
$$

where $\sigma$ is the intersection of $\Delta$ with its left vertical supporting line.

The proof of Theorem 1 is given in Section 4. It mainly follows the argument of [2], [3], where a description of the first order degenerations and respective bifurcations of simple parameterizations of tropical curves in count are given. Notice that Theorem 1 can also be proved via the study of non-parameterized plane tropical curves in the spirit of [8].

3.2. Recursive formula for multi-component invariants. Denote by $\theta_{k}$ the element in $\ell$ whose $k$-th term is equal to 1 and all the other terms are equal to 0 . For $\alpha, \alpha^{\prime} \in \mathcal{C}, \alpha \leq \alpha^{\prime}$, put

$$
\left(\begin{array}{c}
\alpha^{\prime} \\
\alpha
\end{array}\right)=\prod_{i=1}^{\infty}\left(\begin{array}{c}
\alpha_{i}^{\prime} \\
\alpha_{i}
\end{array}\right)
$$

Extend the definition of the multi-component relative tropical Welschinger invariants to the degenerate case $\Delta=\sigma$ in the following way. If $\Delta$ is a point, then put

$$
\mathrm{W}(\Delta,(0),(0), g)= \begin{cases}1, & \text { if } g=0, \\ 0, & \text { otherwise }\end{cases}
$$

If $\Delta$ is a vertical segment, then put $\mathrm{W}(\Delta, \alpha, \beta, g)=1$ for $\alpha+\beta=(|\sigma|), g=1-|\sigma|$, and put $\mathrm{W}(\Delta, \alpha, \beta, g)=0$, in all other cases. A number $\mathrm{W}(\Delta, \alpha, \beta, g)$ such that $\Delta=\sigma$ will be referred to as an initial value.

In addition, put $\mathrm{W}(\Delta, \alpha, \beta, g)=0$ whenever $\Delta$ is nondegenerate and $r \leq\|\alpha\|$.

Given a convex lattice polygon $\Delta$ and a cooriented straight line $\vec{s}$ of slope $0,-1$ or $\infty$, take the supporting straight line $L_{\vec{s}}$ of $\Delta$ such that $L_{\vec{s}}$ is parallel to $\vec{s}$, and $\Delta$ is contained in the half-plane defined by the coorientation.

Then the $\vec{s}$-peeling $l_{\vec{s}}(\Delta)$ of $\Delta$ is the convex hull of $\left(\Delta \cap \mathbb{Z}^{2}\right) \backslash L_{\vec{s}}$.

Introduce the set $\Xi$ formed by the empty set, the lattice points, the lattice vertical segments, and the convex lattice left-nondegenerate polygons $\Delta$ such that a primitive integer normal vector of any face of $\Delta$ belongs to the set

$$
\{(1,0),(-1,0),(0,1),(0,-1),(1,1),(-1,-1)\} \text {. }
$$

Remark 1. Any nondegenerate polygon in $\Xi$ defines a toric unnodal Del Pezzo surface and an ample divisor on it. The set $\Xi$ is closed with respect to the Minkowski sum 
and any $\vec{s}$-peeling $\Delta \mapsto l_{\vec{s}}(\Delta)$, where $\vec{s}$ is a cooriented straight vertical line. Furthermore, if the Minkowski sum of several convex lattice left-nondegenerate polygons is a polygon in $\Xi$, then all the summands are in $\Xi$.

Let $\Delta \in \Xi$ be a nondegenerate polygon. Denote by $\vec{\infty}$ (respectively, $\overrightarrow{0}, \overrightarrow{-1}$ ) a vertical line (respectively, a horizontal line, a line of slope -1 ) cooriented by the vector $(1,0)$ (respectively, $(0,-1),(1,1)$ ). Denote by $L_{\overrightarrow{0}}$ (respectively, $L_{-1}$ ) the support straight line of $\Delta$ such that $L_{\overrightarrow{0}}$ (respectively, $L_{-1}$ ) is parallel to $\overrightarrow{0}$ (respectively, $\overrightarrow{-1}$ ), and $\Delta$ is contained in the half-plane defined by the coorientation. We say that $\Delta$ is $\overrightarrow{0}$-nondegenerate (respectively, $\overrightarrow{-1}$-nondegenerate), if the intersection $d$ of $\Delta$ with $L_{\overrightarrow{0}}$ (respectively, $L_{-1}$ ) is not a vertex, and one of the edges neighboring to the edge $d$ is of slope -1 (respectively, 0 ). Consider a subset $T$ of $\{\overrightarrow{0}, \overrightarrow{-1}\}$. The $T$-peeling $l_{\top}(\Delta)$ of $\Delta$ is the result of the consecutive $\vec{s}$-peelings of $l_{\vec{\infty}}(\Delta)$, where $\vec{s}$ runs over the elements of $\boldsymbol{T}$ (note that in the case $\boldsymbol{T}=\{\overrightarrow{0}, \overrightarrow{-1}\}$, one has $l_{-1}\left(l_{\overrightarrow{0}}\left(l_{\vec{\infty}}(\Delta)\right)\right)=l_{\overrightarrow{0}}\left(l_{-1}\left(l_{\vec{\infty}}(\Delta)\right)\right)$ since $l_{\vec{\infty}}(\Delta)$ is left-nondegenerate). The set $T$ is called $\Delta$-admissible if for any $\vec{s} \in T$ the polygon $\Delta$ is $\vec{s}$-nondegenerate, and the polygon $l_{T}(\Delta)$ is either left-nondegenerate or a point. Note that if $T$ is $\Delta$-admissible, then $l_{\top}(\Delta) \in \Xi$.

Theorem 3. Let $\Delta \in \Xi$ be a nondegenerate polygon, and $\sigma$ the intersection of $\Delta$ with its left vertical supporting line. Then, for any $\alpha, \beta \in \mathcal{C}$ such that $J \alpha+J \beta=|\sigma|$, and any integer $g$, one has

$$
\begin{aligned}
\mathrm{W}(\Delta, \alpha, \beta, g)= & \sum_{\substack{k \geq 1 \\
\beta_{k}>0}} \mathrm{~W}\left(\Delta, \alpha+\theta_{k}, \beta-\theta_{k}, g\right) \\
& +\sum_{\top, \alpha^{\prime}, \beta^{\prime}, g^{\prime}}\left(\begin{array}{c}
\alpha \\
\alpha^{\prime}
\end{array}\right)\left(\begin{array}{c}
\beta^{\prime} \\
\beta
\end{array}\right) \mathrm{W}\left(l_{\top}(\Delta), \alpha^{\prime}, \beta^{\prime}, g^{\prime}\right),
\end{aligned}
$$

where the latter sum in (2) runs over the quadruples $T, \alpha^{\prime}, \beta^{\prime}, g^{\prime}$ satisfying the following conditions:

$$
\begin{gathered}
ד \subset\{\overrightarrow{0}, \overrightarrow{-1}\} \text { is } \Delta \text {-admissible, } \quad \alpha^{\prime}, \beta^{\prime} \in \mathcal{C}, \quad g^{\prime} \in \mathbb{Z}, \quad \alpha^{\prime} \leq \alpha, \quad \beta \leq \beta^{\prime}, \\
(\alpha, \beta) \neq\left(\alpha^{\prime}, \beta^{\prime}\right), \quad J \alpha^{\prime}+J \beta^{\prime}=\left|\sigma^{\prime}\right|, \quad g-g^{\prime}=\left\|\beta^{\prime}-\beta\right\|-1,
\end{gathered}
$$

$\sigma^{\prime}$ being the intersection of $l_{\top}(\Delta)$ with its left vertical supporting line.

The proof of Theorem 3 basically follows the lines of the proof of Theorem 4.3 from [3], and is presented in Section 5.

Remark 2. The initial values for $\mathrm{W}(\Delta, \alpha, \beta, g)$ (i.e., the numbers $\mathrm{W}(\Delta, \alpha, \beta, g)$ in the case $\Delta=\sigma$ ) and the recursive formula given in (2) determine all the numbers $\mathrm{W}(\Delta, \alpha, \beta, g), \Delta \in \Xi$. 
Formula (2) can be seen as a real analogue of the Caporaso-Harris formula [1], Theorem 1.1, and of its generalizations proposed by R. Vakil [9]. The CaporasoHarris formula contains extra coefficients and extra terms with respect to formula (2). Comparing two formulas, one should take into account that a term indexed by $(\alpha, \beta)$ in formula (2) is an analog of the term indexed by $(j(\alpha), j(\beta))$ of the CaporasoHarris formula, where $j: \ell \rightarrow \ell$ is the injection associating to a sequence $\alpha=$ $\left(\alpha_{1}, \alpha_{2}, \alpha_{3}, \ldots\right) \in \mathcal{C}$ the sequence $\left(\alpha_{1}, 0, \alpha_{2}, 0, \alpha_{3}, 0, \ldots\right)$. In other words, in formula (2) we do not consider the tropical analogs of curves which have even order intersections with the fixed straight line. Notice also that the Caporaso-Harris formula contains as a parameter the number of double points instead of the genus. This difference is not essential, since the genus determines the number of double points and vice versa.

3.3. Recursive formula for irreducible invariants. For $\alpha, \alpha^{(1)}, \ldots, \alpha^{(s)} \in \mathcal{C}$, $\alpha \geq \alpha^{(1)}+\cdots+\alpha^{(s)}$, put

$$
\left(\begin{array}{c}
\alpha \\
\alpha^{(1)}, \ldots, \alpha^{(s)}
\end{array}\right)=\prod_{i=1}^{\infty} \frac{\alpha_{i} !}{\alpha_{i}^{(1)} ! \cdots \cdots \alpha_{i}^{(s)} !\left(\alpha_{i}-\sum_{k} \alpha_{i}^{(k)}\right) !} .
$$

Introduce the set $\delta$ of the 4-tuples $(\Delta, \alpha, \beta, g)$ formed by a polygon $\Delta \in \Xi$, elements $\alpha$ and $\beta$ in $\ell$ such that $J \alpha+J \beta=|\sigma|$, where $\sigma$ is the intersection of $\Delta$ with its left vertical supporting line, and an integer $g$. Define in $\delta$ the following operation:

$$
(\Delta, \alpha, \beta, g)+(\tilde{\Delta}, \tilde{\alpha}, \tilde{\beta}, \tilde{g})=(\Delta+\tilde{\Delta}, \alpha+\tilde{\alpha}, \beta+\tilde{\beta}, g+\tilde{g}-1) .
$$

We extend the definition of the irreducible relative tropical Welschinger invariants to the degenerate case $\Delta=\sigma$ in the following way. If $\Delta=\sigma$, put $\mathrm{W}^{\text {irr }}(\Delta, \alpha, \beta, g)=$ 1 for $g=0, \alpha+\beta=(|\sigma|)$, and $|\sigma| \leq 1$, and $\operatorname{put}^{\mathrm{irr}}(\Delta, \alpha, \beta, g)=0$, in all other cases. A number $\mathrm{W}^{\mathrm{irr}}(\Delta, \alpha, \beta, g)$ such that $\Delta=\sigma$ will be referred to as anitial value.

In addition, put $\mathrm{W}^{\text {irr }}(\Delta, \alpha, \beta, g)=0$ whenever $g<0$.

The irreducible relative tropical Welschinger invariants satisfy a recursive formula which is similar to the Caporaso-Harris formula for irreducible relative GromovWitten invariants (see [1], Section 1.4).

Theorem 4. Let $\Delta \in \Xi$ and $\sigma$ be as in Theorem 3. Then, for any $\alpha, \beta \in \mathcal{C}$ such that $J \alpha+J \beta=|\sigma|$, and any integer $g \geq 0$, one has

$$
\begin{aligned}
& \mathrm{W}^{\mathrm{irr}}(\Delta, \alpha, \beta, g)=\sum_{\substack{k \geq 1 \\
\beta_{k}>0}} \mathrm{~W}^{\mathrm{irr}}\left(\Delta, \alpha+\theta_{k}, \beta-\theta_{k}, g\right) \\
& \quad+\sum\left(\begin{array}{c}
\alpha \\
\alpha^{(1)}, \ldots, \alpha^{(m)}
\end{array}\right) \frac{n !}{n_{1} ! \ldots n_{m} !} \prod_{i=1}^{m}\left(\left(\begin{array}{c}
\beta^{(i)} \\
\tilde{\beta}^{(i)}
\end{array}\right) \mathrm{W}^{\mathrm{irr}}\left(\Delta^{(i)}, \alpha^{(i)}, \beta^{(i)}, g^{(i)}\right)\right),
\end{aligned}
$$


where

$n=|\partial \Delta|-|\sigma|+\|\beta\|+g-2, \quad n_{i}=\left|\partial \Delta^{(i)}\right|-\left|\sigma^{(i)}\right|+\left\|\beta^{(i)}\right\|+g^{(i)}-1, \quad i=1, \ldots, m$, and the latter sum in (4) is taken

- over all $\Delta$-admissible sets $\rceil \subset\{\overrightarrow{0}, \overrightarrow{-1}\}$,

- over all splittings

$$
\left(l_{\top}(\Delta), \alpha^{\prime}, \beta^{\prime}, g^{\prime}\right)=\sum_{i=1}^{m}\left(\Delta^{(i)}, \alpha^{(i)}, \beta^{(i)}, g^{(i)}\right)
$$

in 8 , where

$$
\begin{gathered}
\alpha^{\prime}, \beta^{\prime} \in \mathcal{C}, \quad g^{\prime} \in \mathbb{Z}, \quad \alpha^{\prime} \leq \alpha, \quad \beta \leq \beta^{\prime}, \\
J \alpha^{\prime}+J \beta^{\prime}=\left|\sigma^{\prime}\right|, \quad g-g^{\prime}=\left\|\beta^{\prime}-\beta\right\|-1,
\end{gathered}
$$

$\sigma^{\prime}$ being the intersection of $l_{\top}(\Delta)$ with its left vertical supporting line,

- over all splittings

$$
\beta^{\prime}=\beta+\sum_{i=1}^{m} \tilde{\beta}^{(i)}, \quad\left\|\tilde{\beta}^{(i)}\right\|>0, i=1, \ldots, m,
$$

satisfying the restriction $\beta^{(i)} \geq \tilde{\beta}^{(i)}, i=1, \ldots, m$,

and factorized by simultaneous permutations in the both splittings.

Remark 3. In the case $g=0$, the right-hand side of formula (4) reduces to the terms with $g^{(i)}=0$ and $\left\|\tilde{\beta}^{(i)}\right\|=1$.

The proof of Theorem 4 is a slight modification of the proof of Theorem 3, and we indicate this modification at the end of Section 5.

The initial values for $\mathrm{W}^{\text {irr }}(\Delta, \alpha, \beta, g)$ and formula (4) determine all the numbers $\mathrm{W}^{\mathrm{irr}}(\Delta, \alpha, \beta, g)$.

Corollary 4. Let $\Delta_{1}$ and $\Delta_{2}$ be two nondegenerate polygons in $\Xi$ such that $\Delta_{2} \subset$ $\Delta_{1}$. Denote by $\Sigma_{i}$ and $D_{i}, i=1,2$, the toric Del Pezzo surface equipped with its tautological real structure and the ample divisor on $\Sigma_{i}$ which are defined by $\Delta_{i}$. Then

$$
\mathrm{W}\left(\Sigma_{1}, D_{1}\right) \geq \mathrm{W}\left(\Sigma_{2}, D_{2}\right) .
$$

If, in addition, the number of interior integer points of $\Delta_{1}$ is greater than the number of interior integer points of $\Delta_{2}$ (i.e., the genus of a generic member of the linear system $\left|D_{1}\right|$ is greater than the genus of a generic member of $\left.\left|D_{2}\right|\right)$, then

$$
\mathrm{W}\left(\Sigma_{1}, D_{1}\right)>\mathrm{W}\left(\Sigma_{2}, D_{2}\right) .
$$


Corollary 5 (cf. [5], Theorem 1.3). Let $\Sigma$ be a toric unnodal Del Pezzo surface equipped with its tautological real structure, and $D \subset \Sigma$ an ample divisor. Then $\mathrm{W}(\Sigma, D)>0$.

Proof of Corollaries 4 and 5. Since $\Delta_{2} \subset \Delta_{1}$, the polygon $\Delta_{2}$ can be obtained from $\Delta_{1}$ by a sequence of peelings. Thus, it is sufficient to treat the case when $\Delta_{2}$ is the result of an $\vec{s}$-peeling of $\Delta_{1}$. Without loss of generality, we can assume that $\vec{s}$ is the left vertical supporting line of $\Delta_{2}$ cooriented so that $\Delta_{2}$ is contained in the half-plane defined by the coorientation. Denote by $\sigma^{\prime}$ the intersection of $\vec{s}$ with $\Delta_{2}$, and by $\sigma$ the intersection of $\Delta_{1}$ with its left vertical supporting line. If $\sigma$ is a point, then $\mathrm{W}\left(\Sigma_{1}, D_{1}\right)=\mathrm{W}\left(\Sigma_{2}, D_{2}\right)$. Assume that $\sigma$ is a nondegenerate segment. One has

$$
\mathrm{W}\left(\Sigma_{1}, D_{1}\right)=\mathrm{W}^{\mathrm{irr}}\left(\Delta_{1}, 0,(|\sigma|), 0\right), \quad \mathrm{W}\left(\Sigma_{2}, D_{2}\right)=\mathrm{W}^{\mathrm{irr}}\left(\Delta_{2}, 0,\left(\left|\sigma^{\prime}\right|\right), 0\right) .
$$

The absolute value of the difference $|\sigma|-\left|\sigma^{\prime}\right|$ is at most 1 . According to Theorem 4 ,

- if $\left|\sigma^{\prime}\right|=|\sigma|+1$, then

$$
\mathrm{W}^{\mathrm{irr}}\left(\Delta_{1}, 0,(|\sigma|), 0\right) \geq\left|\sigma^{\prime}\right| \cdot \mathrm{W}^{\mathrm{irr}}\left(\Delta_{2}, 0,\left(\left|\sigma^{\prime}\right|\right), 0\right) ;
$$

- if $\left|\sigma^{\prime}\right|=|\sigma|$, then

$$
\begin{aligned}
\mathrm{W}^{\mathrm{irr}}\left(\Delta_{1}, 0,(|\sigma|), 0\right) & \geq \mathrm{W}^{\mathrm{irr}}\left(\Delta_{1},(1),(|\sigma|-1), 0\right) \\
& \geq\left|\sigma^{\prime}\right| \cdot \mathrm{W}^{\mathrm{irr}}\left(\Delta_{2}, 0,\left(\left|\sigma^{\prime}\right|\right), 0\right) ;
\end{aligned}
$$

- if $\left|\sigma^{\prime}\right|=|\sigma|-1$, then

$$
\begin{aligned}
\mathrm{W}^{\mathrm{irr}}\left(\Delta_{1}, 0,(|\sigma|), 0\right) & \geq \mathrm{W}^{\mathrm{irr}}\left(\Delta_{1},(1),(|\sigma|-1), 0\right) \\
& \geq \mathrm{W}^{\mathrm{irr}}\left(\Delta_{1},(2),(|\sigma|-2), 0\right) \\
& \geq\left|\sigma^{\prime}\right| \cdot \mathrm{W}^{\mathrm{irr}}\left(\Delta_{2}, 0,\left(\left|\sigma^{\prime}\right|\right), 0\right) .
\end{aligned}
$$

Thus, in all the three cases,

$$
\mathrm{W}^{\mathrm{irr}}\left(\Delta_{1}, 0,(|\sigma|), 0\right) \geq\left|\sigma^{\prime}\right| \cdot \mathrm{W}^{\mathrm{irr}}\left(\Delta_{2}, 0,\left(\left|\sigma^{\prime}\right|\right), 0\right) .
$$

This proves the inequality $\mathrm{W}\left(\Sigma_{1}, D_{1}\right) \geq \mathrm{W}\left(\Sigma_{2}, D_{2}\right)$. Since, in addition, we have $\mathrm{W}\left(\Sigma_{2}, D_{2}\right)=1$ whenever $\Delta_{2}$ does not have interior integer points, we obtain positivity of the invariants $\mathrm{W}(\Sigma, D)$.

If the number of interior integer points of $\Delta_{1}$ is greater than the number of interior integer points of $\Delta_{2}$, then $\left|\sigma^{\prime}\right| \geq 2$, and inequality (7) implies that $\mathrm{W}\left(\Sigma_{1}, D_{1}\right)>$ $\mathrm{W}\left(\Sigma_{2}, D_{2}\right)$.

Corollary 6. The first six terms of the sequence $\mathrm{W}\left(\mathbb{P}^{2}, d \mathbb{P}^{1}\right)$ are as follows:

$$
\begin{gathered}
\mathrm{W}\left(\mathbb{P}^{2}, \mathbb{P}^{1}\right)=\mathrm{W}\left(\mathbb{P}^{2}, 2 \mathbb{P}^{1}\right)=1, \quad \mathrm{~W}\left(\mathbb{P}^{2}, 3 \mathbb{P}^{1}\right)=8, \quad \mathrm{~W}\left(\mathbb{P}^{2}, 4 \mathbb{P}^{1}\right)=240, \\
\mathrm{~W}\left(\mathbb{P}^{2}, 5 \mathbb{P}^{1}\right)=18264, \quad \mathrm{~W}\left(\mathbb{P}^{2}, 6 \mathbb{P}^{1}\right)=2845440 .
\end{gathered}
$$




\section{Invariance of tropical Welschinger numbers}

4.1. Moduli spaces of parameterized marked tropical curves. Let $\Delta, \alpha, \beta, g$, and $r$ be as in Section 3.1. A parameterized marked tropical curve $(\Gamma, w, h, \boldsymbol{P})$ of degree $\Delta^{\alpha, \beta}$ is a parameterized plane tropical curve $(\Gamma, w, h)$ of degree $\Delta^{\alpha, \beta}$ equipped with a sequence $\boldsymbol{P}$ of $r$ distinct points in $\Gamma$ such that

- $\boldsymbol{P}=\boldsymbol{P}^{\mathrm{b}} \cup \boldsymbol{P}^{\sharp}$ starts with a sequence $\boldsymbol{P}^{\mathrm{b}}$ of some univalent vertices of $\Gamma$ and terminates with a sequence $\boldsymbol{P}^{\sharp}$ whose points are not univalent vertices of $\Gamma$,

- the number of points in $\boldsymbol{P}^{b}$ is equal to $\|\alpha\|$,

- the weight of the ends of $\Gamma$ merging to the points

$$
P_{k} \in P^{b}, \quad \sum_{j<i} \alpha_{j}<k \leq \sum_{j \leq i} \alpha_{j},
$$

is $2 i-1, i \geq 1$,

- those points of $\boldsymbol{P}^{\sharp}$ that coincide with vertices of $\Gamma$ can be pushed inside the adjacent edges in order to transform $\boldsymbol{P}$ to a set $\widetilde{\boldsymbol{P}}$ such that the components of $\bar{\Gamma} \backslash \widetilde{\boldsymbol{P}}$ have no loops and each of them contains at most one univalent vertex (in particular, the components of $\bar{\Gamma} \backslash \boldsymbol{P}$ have no loops and each of them contains at most one univalent vertex).

We sometimes call such a sequence $\boldsymbol{P}$ a configuration. The elements of $\boldsymbol{P}$ are called marked points.

Lemma 7. Let $(\Gamma, w, h, \boldsymbol{P})$ be a parameterized marked tropical curve such that no point of $\boldsymbol{P}$ coincides with a non-univalent vertex of $\Gamma$. Then any connected component of $\bar{\Gamma} \backslash \boldsymbol{P}$ contains exactly one univalent vertex.

Proof. Identifying all the points of $\mathcal{V} \subset \bar{\Gamma}$, we obtain a graph whose first Betti number is equal to $r-\|\alpha\|$. The complement of $\boldsymbol{P}^{\sharp}$ in this graph is a tree, and the statement follows.

Two parameterized marked tropical curves $(\Gamma, w, h, \boldsymbol{P})$ and $\left(\Gamma^{\prime}, w^{\prime}, h^{\prime}, \boldsymbol{P}^{\prime}\right)$ of the same degree $\Delta^{\alpha, \beta}$ are called isomorphic if there is a homeomorphism $\varphi: \Gamma \rightarrow \Gamma^{\prime}$ such that $\varphi(\boldsymbol{P})=\boldsymbol{P}^{\prime}$, and $w(E)=w^{\prime}(\varphi(E))$ for any $E \in \Gamma^{1}$. Two parameterized marked tropical curves $(\Gamma, w, h, \boldsymbol{P})$ and $\left(\Gamma^{\prime}, w^{\prime}, h^{\prime}, \boldsymbol{P}^{\prime}\right)$ of the same degree $\Delta^{\alpha, \beta}$ have the same combinatorial type, if there is a homeomorphism $\varphi: \Gamma \rightarrow \Gamma^{\prime}$ such that

- for any $V \in \Gamma_{0}^{0}$ and any edge $E$ adjacent to $V$, the vectors $u(V, E)$ and $u(\varphi(V), \varphi(E))$ coincide, where $u(V, E)$ (respectively, $u(\varphi(V), \varphi(E))$ ) is the primitive integer vector starting at $h(V)$ (respectively, $\left.h^{\prime}(\varphi(V))\right)$ and directed along $h(E)$ (respectively, $h^{\prime}(\varphi(E))$ ), 
- $w(E)=w^{\prime}(\varphi(E))$ for any $E \in \Gamma^{1}$,

- if a point $P_{i} \in \boldsymbol{P}$ belongs to an edge $E$ (respectively, coincides with a vertex $V$ ) of $\Gamma$, then the point $P_{i}^{\prime}$ belongs to the edge $\varphi(E)$ (respectively, coincides with the vertex $\varphi(V))$.

Let $\Lambda_{\Delta, \alpha, \beta, g}$ be the set of all the combinatorial types of parameterized marked tropical curves $(\Gamma, w, h, \boldsymbol{P})$ of degree $\Delta^{\alpha, \beta}$ such that $b_{1}(\Gamma) \leq g$ and $\boldsymbol{P}^{\sharp}$ contains at least $g-b_{1}(\Gamma)$ points coinciding with vertices of $\Gamma$. For any $\lambda \in \Lambda_{\Delta, \alpha, \beta, g}$ denote by $\mathcal{M}_{\Delta, \alpha, \beta, g}^{\lambda}$ the set of the isomorphism classes of parameterized marked tropical curves of combinatorial type $\lambda$. One can encode the elements $(\Gamma, w, h, \boldsymbol{P})$ of $\mathcal{M}_{\Delta, \alpha, \beta, g}^{\lambda}$ by

(i) the lengths of images under $h$ of all the edges $E \in \Gamma^{1} \backslash \Gamma_{\infty}^{1}$,

(ii) the position of $h(V) \in \widehat{\mathbb{R}}^{2}$ for some vertex $V \in \Gamma_{0}^{0}$,

(iii) the coordinates of the points of $h\left(\boldsymbol{P}^{b}\right)$ on $L_{-\infty}$, and

(iv) the distances of the points of $h\left(\boldsymbol{P}^{\sharp}\right)$ to the images under $h$ of certain chosen vertices of the edges of $\Gamma$ which contain the points of $\boldsymbol{P}^{\sharp}$.

These parameters are called graphic coordinates. The graphic coordinates described in the item (iv) are called marked. For a given $\lambda$, graphic coordinates are subject to finitely many linear equalities and inequalities and identify $\mathcal{M}_{\Delta, \alpha, \beta, g}^{\lambda}$ with the relative interior of a convex polyhedron in an affine space. We call $\mathcal{M}_{\Delta, \alpha, \beta, g}^{\lambda, \alpha, \beta}$ the moduli space of parameterized marked tropical curves of combinatorial type $\lambda$.

A combinatorial type $\lambda^{\prime} \in \Lambda_{\Delta, \alpha, \beta, g}$ is a degeneration of $\lambda \in \Lambda_{\Delta, \alpha, \beta, g}$ if graphic coordinates on $\mathcal{M}_{\Delta, \alpha, \beta, g}^{\lambda}$ and $\mathcal{M}_{\Delta, \alpha, \beta, g}^{\lambda^{\prime}}$ can be chosen in such a way that $\mathcal{M}_{\Delta, \alpha, \beta, g}^{\lambda^{\prime}}$ becomes the intersection of $\mathcal{M}_{\Delta, \alpha, \beta, g}^{\lambda}$ with some coordinate hyperplanes in the following sense: the non-zero coordinates of any point of this intersection are the chosen graphic coordinates of the corresponding point of $\mathcal{M}_{\Delta, \alpha, \beta, g}^{\lambda^{\prime}}$.

For each combinatorial type $\lambda$ contained in $\Lambda_{\Delta, \alpha, \beta, g}$ choose graphic coordinates on $\mathcal{M}_{\Delta, \alpha, \beta, g}^{\lambda}$. Denote by $\overline{\mathcal{P}}^{\lambda}$ the corresponding polyhedron and by $\mathcal{P}^{\lambda}$ its relative interior. If $\lambda^{\prime}$ is a degeneration of $\lambda$, then for any choice of graphic coordinates on $\mathcal{M}_{\Delta, \alpha, \beta, g}^{\lambda}$ there exists a unique choice of graphic coordinates on $\mathcal{M}_{\Delta, \alpha, \beta, g}^{\lambda^{\prime}}$ such that $\mathcal{M}_{\Delta, \alpha, \beta, g}^{\lambda^{\prime}, \beta, g}$ becomes the intersection of $\mathcal{M}_{\Delta, \alpha, \beta, g}^{\lambda}$ with some coordinate hyperplanes. Denote by $f_{\lambda^{\prime}, \lambda}$ the affine map identifying this intersection with $\mathcal{P}^{\lambda^{\prime}}$.

Proposition 8. For any combinatorial type $\lambda \in \Lambda_{\Delta, \alpha, \beta, g}$ and any face $F$ of the polyhedron $\overline{\mathcal{P}}^{\lambda}$, there exists a degeneration $\lambda^{\prime} \in \Lambda_{\Delta, \alpha, \beta, g}$ such that $f_{\lambda^{\prime}, \lambda}^{-1}\left(\mathcal{P}^{\lambda^{\prime}}\right)=F$.

Proof. Pick a parameterized marked tropical curve $(\Gamma, w, h, \boldsymbol{P})$ of combinatorial type $\lambda$. The map $h$ induces a metric on $\Gamma$, and thus, an affine structure on it. Any point in $\mathcal{M}_{\Delta, \alpha, \beta, g}^{\lambda}$ has a representative $(\Gamma, w, \tilde{h}, \widetilde{\boldsymbol{P}})$ such that $\tilde{h}$ is affine-linear on 
any segment that contains no marked point and no vertex of $\Gamma$. Let $p \in F$ be the limit of a sequence of points in $\mathcal{P}^{\lambda}$. The sequence of corresponding maps $\tilde{h}$ converges to a map $\Gamma \rightarrow \widehat{\mathbb{R}}^{2}$ which is a composition of a quotient map $\psi$ from $\Gamma$ to a certain graph $\Gamma^{\prime}$ and an embedding $h^{\prime}: \Gamma^{\prime} \rightarrow \widehat{\mathbb{R}}^{2}$. The sequence of configurations $\widetilde{\boldsymbol{P}}$ converges to a configuration $\overline{\boldsymbol{P}}$ of points of $\Gamma$. Descending $w$ and $\overline{\boldsymbol{P}}$ to $\Gamma^{\prime}$, one obtains a parameterized marked tropical curve $\left(\Gamma^{\prime}, w \circ \psi^{-1}, h^{\prime}, \psi(\overline{\boldsymbol{P}})\right)$ together with graphical coordinates identifying it with $p$. The combinatorial type of $\left(\Gamma^{\prime}, w \circ \psi^{-1}, h^{\prime}, \psi(\overline{\boldsymbol{P}})\right)$ is a degeneration of $\lambda$.

For any $\lambda \in \Lambda(\Delta, \alpha, \beta, g)$, denote by $\mathcal{Q}^{\lambda}$ the projection of $\mathcal{P}^{\lambda}$ on the coordinate subspace spanned by the non-marked graphical coordinate axes.

Lemma 9 (see [7], Proposition 2.23). For any $\lambda \in \Lambda(\Delta, \alpha, \beta, g)$, the dimension of $Q^{\lambda}$ is at most $r$. Moreover, $Q^{\lambda}$ is of dimension $r$ if and only if the curves in $\lambda$ are simply parameterized.

If $(\Gamma, w, h, \boldsymbol{P})$ is a simply parameterized marked tropical curve of degree $\Delta^{\alpha, \beta}$ such that no point in $\boldsymbol{P}^{\sharp}$ is a vertex of $\Gamma$, then the combinatorial type of $(\Gamma, w, h, \boldsymbol{P})$ is called $\sigma$-generic.

Consider the disjoint union $\bigsqcup_{\lambda} \overline{\mathcal{P}}^{\lambda}$, where $\lambda$ runs over all the $\sigma$-generic combinatorial types in $\Lambda_{\Delta, \alpha, \beta, g}$. Let $\mathcal{M}_{\Delta, \alpha, \beta, g}$ be the quotient space of $\coprod_{\lambda} \overline{\mathcal{P}}^{\lambda}$ defined by the gluing maps $f_{\lambda^{\prime}, \lambda_{2}}^{-1} \circ f_{\lambda^{\prime}, \lambda_{1}}$ for all the triples $\left(\lambda_{1}, \lambda_{2}, \lambda^{\prime}\right)$ of combinatorial types such that $\lambda_{1}$ and $\lambda_{2}$ are $\sigma$-generic, and $\lambda^{\prime}$ is a degeneration of $\lambda_{1}$ and $\lambda_{2}$. We identify the sets $\mathcal{M}_{\Delta, \alpha, \beta, g}^{\lambda}$ with $\mathcal{P}^{\lambda}$ and consider them as subspaces in $\mathcal{M}_{\Delta, \alpha, \beta, g}$.

In the case $\alpha=(0)$, the moduli space $\mathcal{M}_{\Delta, \alpha, \beta, g}$ coincides with a moduli space introduced by Gathmann and Markwig [2] (in [2] this space is denoted by $\overline{\mathcal{M}}_{g, \Delta}$, where $\left.\Delta=\Delta^{(0), \beta}\right)$.

4.2. Collar. For a combinatorial type $\lambda \in \Lambda_{\Delta, \alpha, \beta, g}$ of parameterized marked tropical curves $(\Gamma, w, h, \boldsymbol{P})$ denote by $\check{\lambda}$ the combinatorial type of parameterized marked tropical curves $(\Gamma, w, h, \check{\boldsymbol{P}})$ of degree $\Delta^{(0), \alpha+\beta}$ such that the configuration $\check{\boldsymbol{P}}$ is obtained from $\boldsymbol{P}$ by pushing the points of $\boldsymbol{P}^{b}$ inside the adjacent edges of $\Gamma$. If $\lambda$ is $\sigma$-generic, so is $\grave{\lambda}$, and vice versa.

Denote by $\check{\mathcal{M}}_{\Delta, \alpha, \beta, g}$ the union $\bigcup_{\lambda \in \Lambda_{\Delta, \alpha, \beta, g}} \mathcal{M}_{\Delta,(0), \beta, g}^{\check{\lambda}} \subset \mathcal{M}_{\Delta,(0), \beta, g}$. Define a map $\Pi: \check{\mathscr{M}}_{\Delta,(0), \beta, g} \rightarrow \mathcal{M}_{\Delta, \alpha, \beta, g}$ associating to $(\Gamma, w, h, \check{\boldsymbol{P}}) \in \check{\mathcal{M}}_{\Delta,(0), \beta, g}$ the element $(\Gamma, w, h, \boldsymbol{P})$ in $\mathcal{M}_{\Delta, \alpha, \beta, g}$ such that the configuration $\boldsymbol{P}$ is obtained from $\check{\boldsymbol{P}}$ by moving each of the first $\|\alpha\|$ points of $\check{\boldsymbol{P}}$ (all these points belong to left ends of $\Gamma$ ) to the closest univalent vertex of $\Gamma$.

Consider the spaces

$$
\Omega(\Delta, \alpha, \beta, g)=\left(L_{-\infty}\right)^{\|\alpha\|} \times\left(\mathbb{R}^{2}\right)^{r-\|\alpha\|} \subset\left(L_{-\infty}\right)^{\|\alpha\|} \times\left(\widehat{\mathbb{R}}^{2}\right)^{r-\|\alpha\|} \subset\left(\widehat{\mathbb{R}}^{2}\right)^{r},
$$


the map pr: $\left(\widehat{\mathbb{R}}^{2}\right)^{r} \rightarrow\left(L_{-\infty}\right)^{\|\alpha\|} \times\left(\widehat{\mathbb{R}}^{2}\right)^{r-\|\alpha\|}$ replacing the first $\|\alpha\|$ abscissaes by $-\infty$, and the evaluation map

$$
\mathrm{ev}: \mathcal{M}_{\Delta, \alpha, \beta, g} \rightarrow\left(L_{-\infty}\right)^{\|\alpha\|} \times\left(\mathbb{R}^{2}\right)^{r-\|\alpha\|}, \quad \operatorname{ev}(\Gamma, w, h, \boldsymbol{P})=h(\boldsymbol{P}) .
$$

Lemma 10. For any $\lambda \in \Lambda_{\Delta, \alpha, \beta, g}$ the restriction of $\Pi$ to $\overline{\mathcal{P}}^{\check{\lambda}} \cap \check{\mathcal{M}}_{\Delta, \alpha, \beta, g}$ is an affine surjective map to $\overline{\mathcal{P}}^{\lambda}$, and its fibers are relative interiors of convex polyhedra of dimension $\|\alpha\|$. Furthermore, the restrictions of the evaluation maps to $\overline{\mathcal{P}}^{\lambda}$ and $\overline{\mathcal{P}}^{\check{\lambda}}$ are affine, and $\mathrm{ev} \circ \Pi=\mathrm{pr} \circ \mathrm{ev}$.

Proof. The restriction of $\Pi$ to $\mathcal{P}^{\check{\lambda}}$ is an affine map to $\mathcal{P}^{\lambda}$. Furthermore, $\Pi$ is continuous (cf. proof of Proposition 8). Repeating the construction of $\check{\lambda}$ out of $\lambda$ one can show that the restriction of $\Pi$ to $\mathcal{P}^{\check{\lambda}}$ is surjective onto $\mathcal{P}^{\lambda}$, and each of its fibers is the product of $\|\alpha\|$ open rays. The last statement of the lemma is straightforward.

\subsection{Zero and one}

Lemma 11. For any combinatorial type $\lambda \in \Lambda_{\Delta, \alpha, \beta, g}$, the dimension of $\mathcal{M}_{\Delta, \alpha, \beta, g}^{\lambda}$ is at most $2 r-\|\alpha\|$ (recall that $r=|\partial \Delta|-|\sigma|+\|\alpha\|+\|\beta\|+g-1)$, and $\operatorname{dim} \mathcal{M}_{\Delta, \alpha, \beta, g}^{\lambda}=2 r-\|\alpha\|$ if and only if $\lambda$ is $\sigma$-generic.

Proof. The statement immediately follows from Lemma 9.

Lemma 12. For any $\sigma$-generic combinatorial type $\lambda$,

(i) the evaluation map ev restricts to a bijection between $\mathcal{M}_{\Delta, \alpha, \beta, g}^{\lambda}$ and the interior of a full dimensional convex polyhedron in $\left(L_{-\infty}\right)^{\|\alpha\|} \times\left(\mathbb{R}^{2}\right)^{r-\|\alpha\|}$, and

(ii) any two parameterized marked tropical curves of combinatorial type $\lambda$ have the same Welschinger multiplicity.

Proof. Pick a $\sigma$-generic combinatorial type $\lambda \in \Lambda_{\Delta, \alpha, \beta, g}$. Due to Lemma 10, the restriction ev ${ }^{\lambda}$ of ev to $\mathcal{P}^{\lambda}$ is affine. Furthermore, according to [2], Proposition 4.2, (cf. [7]) the restriction ev $\mathrm{ev}^{\check{\lambda}}$ of ev to $\mathcal{P}^{\check{\lambda}}$ is injective. The equality ev $\circ \Pi=\operatorname{pr} \circ \mathrm{ev}$ (see Lemma 10) and the injectivity of ev $\mathrm{v}^{\check{\lambda}}$ imply the injectivity of ev ${ }^{\lambda}$, since if a point in $\operatorname{ev}\left(\mathcal{P}^{\lambda}\right)$ has two distinct inverse images $\left(\Gamma_{1}, w_{1}, h_{1}, \boldsymbol{P}_{1}\right)$ and $\left(\Gamma_{2}, w_{2}, h_{2}, \boldsymbol{P}_{2}\right)$ under ev $\circ \Pi$, then modifying $h_{1}$ and $h_{2}$ (alternatively moving some points of $\boldsymbol{P}_{1}$ and $\boldsymbol{P}_{2}$ ) one gets two distinct points in $\mathcal{P}^{\check{\lambda}}$ with the same image under ev.

The second statement of the lemma immediately follows from the definition of the Welschinger multiplicity. 
The Welschinger multiplicity of the parameterized marked tropical curves of $\sigma$ generic combinatorial type $\lambda$ is denoted by $\mathrm{W}(\lambda)$.

Our goal is to study the complement in $\left(L_{-\infty}\right)^{\|\alpha\|} \times\left(\mathbb{R}^{2}\right)^{r-\|\alpha\|}$ of the union $\bigcup_{\lambda} \operatorname{ev}\left(\mathcal{M}_{\Delta, \alpha, \beta, g}^{\lambda}\right)$, where $\lambda$ runs over all the combinatorial types in $\Lambda_{\Delta, \alpha, \beta, g}$ such that the codimension of $\operatorname{ev}\left(\mathcal{M}_{\Delta, \alpha, \beta, g}^{\lambda}\right)$ in $\left(L_{-\infty}\right)^{\|\alpha\|} \times\left(\mathbb{R}^{2}\right)^{r-\|\alpha\|}$ is at least 2. A combinatorial type $\lambda \in \Lambda_{\Delta, \alpha, \beta, g}$ is called injective codimension 1 if $\mathcal{M}_{\Delta, \alpha, \beta, g}^{\lambda}$ is of codimension 1 in $\mathcal{M}_{\Delta, \alpha, \beta, g}$, and the restriction of ev to $\mathcal{M}_{\Delta, \alpha, \beta, g}^{\lambda}$ is injective. The set of combinatorial types $\lambda \in \Lambda_{\Delta, \alpha, \beta, g}$ such that the codimension of $\operatorname{ev}\left(\mathcal{M}_{\Delta, \alpha, \beta, g}^{\lambda}\right)$ in $\left(L_{-\infty}\right)^{\|\alpha\|} \times\left(\mathbb{R}^{2}\right)^{r-\|\alpha\|}$ is at most 1 consists of

- all $\sigma$-generic combinatorial types in $\Lambda_{\Delta, \alpha, \beta, g}$ (see Lemmas 11 and 12) and

- all the injective codimension 1 combinatorial types in $\Lambda_{\Delta, \alpha, \beta, g}$.

Lemma 13. Let $\lambda \in \Lambda_{\Delta, \alpha, \beta, g}$ be injective codimension 1 . Then $\check{\lambda}$ is injective codimension 1. Furthermore, any element in $\mathcal{M}_{\Delta, \alpha, \beta, g}^{\lambda}$ is represented by a parameterized marked tropical curve $(\Gamma, w, h, \boldsymbol{P})$ such that $b_{1}(\Gamma)=g$ and

(i) either $\Gamma$ is trivalent, and exactly one point of $\boldsymbol{P}^{\sharp}$ is a vertex of $\Gamma$,

(ii) or one of the vertices of $\Gamma$ is four-valent, the other non-univalent vertices of $\Gamma$ are trivalent, and the points of $\boldsymbol{P}^{\sharp}$ are not vertices of $\Gamma$,

(iii) or $\Gamma$ has two four-valent vertices joined by two edges $E_{1}$ and $E_{2}$, the other non-univalent vertices of $\Gamma$ are trivalent, and the points of $\boldsymbol{P}^{\sharp}$ are not vertices of $\Gamma$.

Proof. Since all the fibers of $\Pi$ have the same dimension (see Lemma 10), the codimension of $\mathcal{M}_{\Delta, \alpha, \beta, g}^{\check{\lambda}} \subset \check{\mathcal{M}}_{\Delta, \alpha, \beta, g}$ is equal to 1 . The fibers of pr have the same dimension as the fibers of $\Pi$. Hence, due to ev $\circ \Pi=\mathrm{pr} \circ \mathrm{ev}$, the injectivity of the restriction of ev to $\mathcal{M}_{\Delta, \alpha, \beta, g}^{\lambda}$ implies the injectivity of the restriction of ev to $\mathcal{M}_{\Delta, \alpha, \beta, g}^{\check{\lambda}}$. The second statement of the lemma follows now from [2], Proposition 3.9 and Remark 3.6.

4.4. First bifurcation. Let $\lambda \in \Lambda_{\Delta, \alpha, \beta, g}$ be an injective codimension 1 combinatorial type, and $(\Gamma, w, h, \boldsymbol{P})$ a parameterized marked tropical curve of combinatorial type $\lambda$. Assume that $\Gamma$ is trivalent, and exactly one point of $\boldsymbol{P}^{\sharp}$ is a vertex of $\Gamma$. Denote this vertex by $V$. The last property in the definition of parameterized marked tropical curves implies that exactly two edges adjacent to $V$ are allowed in the following sense: pushing the point which coincides with $V$ to any of these edges creates neither a loop in $\bar{\Gamma} \backslash \boldsymbol{P}$ nor a component of $\bar{\Gamma} \backslash \boldsymbol{P}$ with more than one univalent vertex. Denote the two resulting $\sigma$-generic combinatorial types by $\lambda_{+}$and $\lambda_{-}$. The combinatorial type $\lambda$ is a degeneration of $\lambda_{+}$and $\lambda_{-}$, and no other $\sigma$-generic combinatorial type has $\lambda$ as degeneration. 
Lemma 14. Let $\lambda, \lambda_{+}$, and $\lambda_{-}$be as above. Then $\operatorname{ev}\left(\mathcal{M}_{\Delta, \alpha, \beta, g}^{\lambda_{+}}\right)$and $\operatorname{ev}\left(\mathcal{M}_{\Delta, \alpha, \beta, g}^{\lambda_{-}}\right)$ are on opposite sides of $\operatorname{ev}\left(\mathcal{M}_{\Delta, \alpha, \beta, g}^{\lambda}\right)$.

Proof. According to Lemma 13, the combinatorial type $\check{\lambda}$ is injective codimension 1 . As is shown in [2], case (c) in the proof of Theorem 4.8, the images ev( $\left(\mathcal{M}_{\Delta, \alpha, \beta, d}^{\check{\lambda}_{+}}\right)$ and $\operatorname{ev}\left(\mathcal{M}_{\Delta, \alpha, \beta, d}^{\check{\lambda}_{-}}\right)$of $\mathcal{M}_{\Delta, \alpha, \beta, g}^{\check{\lambda}_{+}}$and $\mathcal{M}_{\Delta, \alpha, \beta, g}^{\check{\lambda}_{-}}$under the evaluation map are on opposite sides of $\operatorname{ev}\left(\mathcal{M}_{\Delta, \alpha, \beta, d}^{\check{\lambda}}\right)$. Thus, the statement of the lemma follows from the relation $\mathrm{ev} \circ \Pi=\mathrm{pr} \circ \mathrm{ev}$ and the fact that $\mathrm{pr}$ is affine.

Lemma 15. Let $\lambda_{1} \lambda_{+}$, and $\lambda_{-}$be as above. Then the Welschinger multiplicities $\mathrm{W}\left(\lambda_{+}\right)$and $\mathrm{W}\left(\lambda_{-}\right)$are equal.

4.5. Third bifurcation. Let $\lambda \in \Lambda_{\Delta, \alpha, \beta, g}$ be an injective codimension 1 combinatorial type, and $(\Gamma, w, h, \boldsymbol{P})$ a parameterized marked tropical curve of combinatorial type $\lambda$. Assume that $\Gamma$ has two four-valent vertices $V$ and $V^{\prime}$ joined by two edges $E_{1}$ and $E_{2}$, the other non-univalent vertices of $\Gamma$ are trivalent, and the points of $\boldsymbol{P}^{\sharp}$ are not vertices of $\Gamma$. A $\sigma$-generic combinatorial type $\tilde{\lambda}$ is a perturbation of $\lambda$ if $\tilde{\lambda}$ is represented by a parameterized marked tropical curve $(\widetilde{\Gamma}, \tilde{w}, \tilde{h}, \widetilde{\boldsymbol{P}})$ such that the graph $\widetilde{\Gamma}$ is obtained from $\Gamma$ replacing each four-valent vertex by two trivalent ones connected by an edge (denote these edges by $E_{V}$ and $E_{V^{\prime}}$, respectively), and there exists a continuous map $\varphi: \widetilde{\Gamma} \rightarrow \Gamma$ satisfying the following properties:

- the image of any vertex of $\widetilde{\Gamma}$ under $\varphi$ is a vertex of $\Gamma$,

- if $E$ is an edge of $\widetilde{\Gamma}$ different from $E_{V}$ and $E_{V^{\prime}}$ and a vertex $W$ is adjacent to $E$, then the image $\varphi(E)$ is an edge of $\Gamma$, the vectors $u(W, E)$ and $u(\varphi(W), \varphi(E))$ coincide,

- $\varphi\left(E_{V}\right)=V$ and $\varphi\left(E_{V^{\prime}}\right)=V^{\prime}$,

- if $E$ is an edge of $\widetilde{\Gamma}$ different from $E_{V}$ and $E_{V^{\prime}}$, then $\tilde{w}(E)=w(\varphi(E))$,

- $\varphi(\widetilde{\boldsymbol{P}})=\boldsymbol{P}$.

Lemma 16. Let $\lambda \in \Lambda_{\Delta, \alpha, \beta, g}$ be as above. Then there are exactly two $\sigma$-generic combinatorial types which admit $\lambda$ as a degeneration. These combinatorial types $\lambda_{+}$

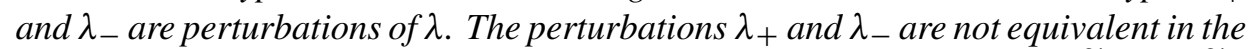
following sense: there is no homeomorphism of their underlying graphs $\widetilde{\Gamma}_{+}$and $\widetilde{\Gamma}_{-}$ which respect the maps $\varphi_{+}$and $\varphi_{-}$(fragments of the graphs $\widetilde{\Gamma}_{+}$and $\widetilde{\Gamma}_{-}$are shown in Figure 2 (a)). Moreover, $\operatorname{ev}\left(\mathcal{M}_{\Delta, \alpha, \beta, g}^{\lambda_{+}}\right)$and $\operatorname{ev}\left(\mathcal{M}_{\Delta, \alpha, \beta, g}^{\lambda_{-}}\right)$are on opposite sides of $\operatorname{ev}\left(\mathcal{M}_{\Delta, \alpha, \beta, g}^{\lambda}\right)$. 

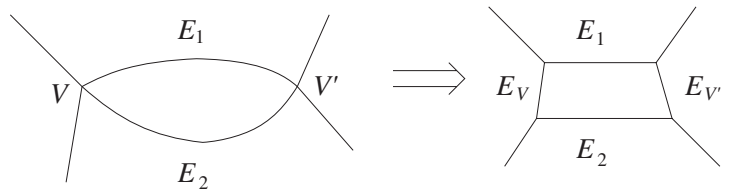

(a)
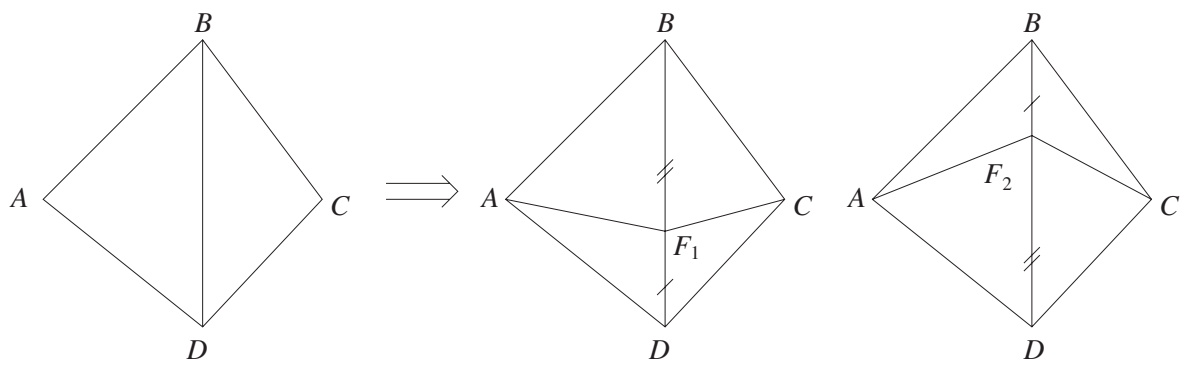

(b)

Figure 2. Third bifurcation.

Proof. According to Lemma 13, the combinatorial type $\check{\lambda}$ is injective codimension 1 . As is shown in [2], case (d) in the proof of Theorem 4.8, there are exactly two $\sigma$-generic combinatorial types $\check{\lambda}_{+}$and $\check{\lambda}_{-}$which admit $\check{\lambda}$ as a degeneration. The underlying graphs of parameterized marked tropical curves representing $\check{\lambda}_{+}$and $\check{\lambda}_{-}$are obtained from $\Gamma$ replacing each four-valent vertex by two trivalent ones as shown on Figure 2 (a), and $\operatorname{ev}\left(\mathcal{M}_{\Delta, \alpha, \beta, d}^{\check{\lambda}_{+}}\right)$and $\operatorname{ev}\left(\mathcal{M}_{\Delta, \alpha, \beta, d}^{\check{\lambda}_{-}}\right)$are on opposite sides of $\operatorname{ev}\left(\mathcal{M}_{\Delta, \alpha, \beta, d}^{\check{\lambda}}\right)$. Thus, the statements of the lemma follow from the relation ev $\circ \Pi=\operatorname{pr} \circ \mathrm{ev}$ and the fact that pr is affine.

Lemma 17. Let $\lambda_{,} \lambda_{+}$, and $\lambda_{-}$be as in Lemma 16. Then $\mathrm{W}\left(\lambda_{+}\right)=\mathrm{W}\left(\lambda_{-}\right)$.

Proof. Consider parameterized marked tropical curves $\left(\widetilde{\Gamma}_{+}, \tilde{w}_{+}, \tilde{h}_{+}, \widetilde{\boldsymbol{P}}_{+}\right)$and $\left(\widetilde{\Gamma}_{-}, \tilde{w}_{-}, \tilde{h}_{-}, \widetilde{\boldsymbol{P}}_{-}\right)$of combinatorial types $\lambda_{+}$and $\lambda_{-}$. The dual subdivisions of plane tropical curves $T_{+}$and $T_{-}$defined by $\left(\widetilde{\Gamma}_{+}, \tilde{w}_{+}, \tilde{h}_{+}, \widetilde{\boldsymbol{P}}_{+}\right)$and $\left(\widetilde{\Gamma}_{-}, \tilde{w}_{-}, \tilde{h}_{-}, \widetilde{\boldsymbol{P}}_{-}\right)$differ by the fragments shown in Figure 2 (b).

The lattice lengths $\left|B F_{1}\right|$ and $\left|F_{2} D\right|$ are equal to $w\left(E_{1}\right)$, and the lattice lengths $\left|F_{1} D\right|$ and $\left|B F_{2}\right|$ are equal to $w\left(E_{2}\right)$. If at least one of the lattice lengths $|A B|,|B C|$, $|C D|,|D A|,\left|B F_{1}\right|=\left|F_{2} D\right|,\left|F_{1} D\right|=\left|B F_{2}\right|$, is even, then $\mathrm{W}\left(T_{+}\right)=\mathrm{W}\left(T_{-}\right)=0$. Assume that all these lengths are odd. Then $\left|F_{1} F_{2}\right|$ is even, and therefore $\left|A F_{1}\right|=$ $\left|A F_{2}\right| \bmod 2$ and $\left|F_{1} C\right|=\left|F_{2} C\right| \bmod 2$. If at least one of the lattice lengths $\left|A F_{1}\right|$ and $\left|F_{1} C\right|$ is even, then $\mathrm{W}\left(T_{+}\right)=\mathrm{W}\left(T_{-}\right)=0$. If $\left|A F_{1}\right|$ and $\left|F_{1} C\right|$ are odd, then the total number $s_{+}$of interior integer points in the triangles $A B F_{1}, A F_{1} D, B C F_{1}$, 
$F_{1} C D$ has the same parity as the number $s_{-}$of interior integer points in the triangles $A B F_{2}, A F_{2} D, B C F_{2}, F_{2} C D$. Hence, $\mathrm{W}\left(T_{+}\right)=(-1)^{s_{+}-s_{-}} \mathrm{W}\left(T_{-}\right)=\mathrm{W}\left(T_{-}\right)$.

\subsection{Second bifurcation}

4.6.1. Preliminaries. Let $\lambda \in \Lambda_{\Delta, \alpha, \beta, g}$ be an injective codimension 1 combinatorial type, and let $(\Gamma, w, h, \boldsymbol{P})$ be a parameterized marked tropical curve of combinatorial type $\lambda$. Assume that one of the vertices of $\Gamma$ is four-valent (denote this vertex by $V$ ), the other non-univalent vertices of $\Gamma$ are trivalent, and the points of $\boldsymbol{P}^{\sharp}$ are not vertices of $\Gamma$. Denote by $E_{1}, E_{2}, E_{3}$, and $E_{4}$ the edges of $\Gamma$ which are adjacent to $V$, and denote by $L_{1}, L_{2}, L_{3}$, and $L_{4}$ the lines containing $h\left(E_{1}\right), h\left(E_{2}\right), h\left(E_{3}\right)$, and $h\left(E_{4}\right)$, respectively.

A $\sigma$-generic combinatorial type $\tilde{\lambda}$ is a perturbation of $\lambda$ if $\tilde{\lambda}$ is represented by a parameterized marked tropical curve $(\widetilde{\Gamma}, \tilde{w}, \tilde{h}, \widetilde{\boldsymbol{P}})$ such that the graph $\widetilde{\Gamma}$ is obtained from $\Gamma$ replacing the vertex $V$ by two trivalent ones connected by an edge (denote this edge by $E_{V}$ ), and there exists a continuous map $\varphi: \widetilde{\Gamma} \rightarrow \Gamma$ satisfying the following properties:

- the image of any vertex of $\widetilde{\Gamma}$ under $\varphi$ is a vertex of $\Gamma$,

- if $E$ is an edge of $\widetilde{\Gamma}$ different from $E_{V}$ and a vertex $W$ is adjacent to $E$, then the image $\varphi(E)$ is an edge of $\Gamma$, and the vector $u(W, E)$ coincides with $u(\varphi(W), \varphi(E))$,

- $\varphi\left(E_{V}\right)=V$,

- if $E$ is an edge of $\widetilde{\Gamma}$ different from $E_{V}$, then $\tilde{w}(E)=w(\varphi(E))$,

- $\varphi(\widetilde{\boldsymbol{P}})=\boldsymbol{P}$.

\subsubsection{Non-degenerate case}

Lemma 18. Let $\lambda \in \Lambda_{\Delta, \alpha, \beta, g}$ be as in Section 4.6.1. Assume that the lines $L_{1}, L_{2}$, $L_{3}$, and $L_{4}$ are pairwise distinct. Then there are exactly three $\sigma$-generic combinatorial types which admit $\lambda$ as a degeneration. These combinatorial types $\lambda_{\times}, \lambda_{+}$, and $\lambda_{-}$are perturbations of $\lambda$ and have the following properties:

- among the edges $\varphi_{\times}^{-1}\left(E_{1}\right), \varphi_{\times}^{-1}\left(E_{2}\right), \varphi_{\times}^{-1}\left(E_{3}\right)$, and $\varphi_{\times}^{-1}\left(E_{4}\right)$, there are two edges, $\varphi_{\times}^{-1}\left(E_{i}\right)$ and $\varphi_{\times}^{-1}\left(E_{j}\right)$, such that the images under $\tilde{h}_{\times}$of their interiors have a common point,

- the edges $\varphi_{+}^{-1}\left(E_{i}\right)$ and $\varphi_{+}^{-1}\left(E_{j}\right)$ have a common vertex,

- the edges $\varphi_{-}^{-1}\left(E_{i}\right)$ and $\varphi_{-}^{-1}\left(E_{j}\right)$ do not have a common vertex. 
Fragments of the graphs $\widetilde{\Gamma}_{\times}, \widetilde{\Gamma}_{+}$, and $\widetilde{\Gamma}_{-}$are shown in Figure 3 (a). The perturbations $\lambda_{\times}, \lambda_{+}$, and $\lambda_{-}$are not equivalent in the same sense as in Lemma 16. Furthermore, $\operatorname{ev}\left(\mathcal{M}_{\Delta, \alpha, \beta, g}^{\lambda_{\times}}\right)$and $\operatorname{ev}\left(\mathcal{M}_{\Delta, \alpha, \beta, g}^{\lambda_{+}}\right)$are on the same side of $\operatorname{ev}\left(\mathcal{M}_{\Delta, \alpha, \beta, g}^{\lambda}\right)$, and $\operatorname{ev}\left(\mathcal{M}_{\Delta, \alpha, \beta, g}^{\lambda^{-}}\right)$is on the opposite side.

Proof. The same arguments as in the proofs of Lemmas 14 and 16 allow one to deduce all the statements of the lemma from [2], case (a) in the proof of Theorem 4.8. To complete the proof of the last statement, consider the dual subdivisions of the tropical curves defined by $\left(\widetilde{\Gamma}_{\times}, \tilde{w}_{\times}, \tilde{h}_{\times}, \widetilde{\boldsymbol{P}}_{\times}\right),\left(\widetilde{\Gamma}_{+}, \tilde{w}_{+}, \tilde{h}_{+}, \widetilde{\boldsymbol{P}}_{+}\right)$, and $\left(\widetilde{\Gamma}_{-}, \tilde{w}_{-}, \tilde{h}_{-}, \widetilde{\boldsymbol{P}}_{-}\right)$ (see Figure $3(\mathrm{~b})$ ), and notice that

$$
\begin{gathered}
\operatorname{Area}(B C F) \cdot \operatorname{Area}(C D F)+\operatorname{Area}(A B D) \cdot \operatorname{Area}(B C D) \\
=\operatorname{Area}(A B C) \cdot \operatorname{Area}(A C D) .
\end{gathered}
$$
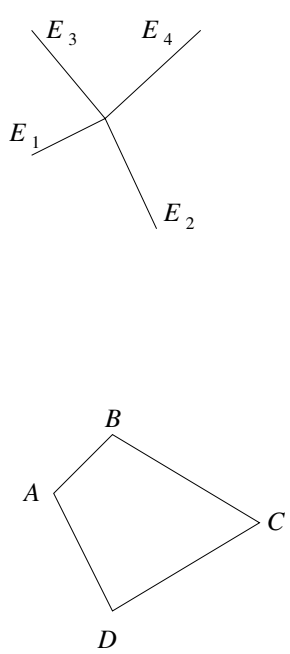

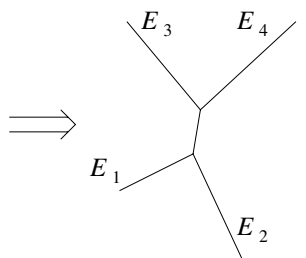

$\tilde{\Gamma}_{-}$

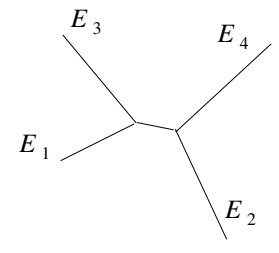

$\tilde{\Gamma}_{+}$

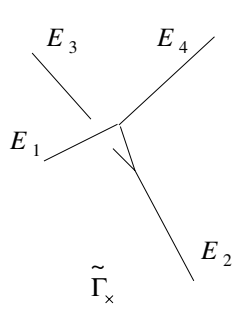

(a)

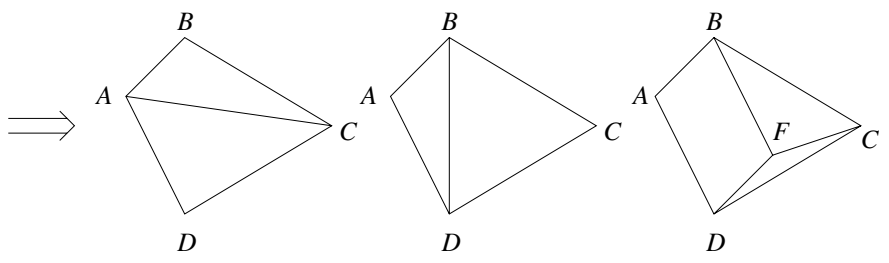

(b)

Figure 3. Non-degenerate second bifurcation.

Lemma 19. Let the combinatorial types $\lambda_{,} \lambda_{\times}, \lambda_{+}$, and $\lambda_{-}$be as in Lemma 18. Then $\mathrm{W}\left(\lambda_{\times}\right)+\mathrm{W}\left(\lambda_{+}\right)=\mathrm{W}\left(\lambda_{-}\right)$.

Proof. Let us consider parameterized marked tropical curves $\left(\widetilde{\Gamma}_{\times}, \tilde{w}_{\times}, \tilde{h}_{\times}, \widetilde{\boldsymbol{P}}_{\times}\right)$, $\left(\widetilde{\Gamma}_{+}, \tilde{w}_{+}, \tilde{h}_{+}, \widetilde{\boldsymbol{P}}_{+}\right)$, and $\left(\widetilde{\Gamma}_{-}, \tilde{w}_{-}, \tilde{h}_{-}, \widetilde{\boldsymbol{P}}_{-}\right)$of combinatorial types $\lambda_{\times}, \lambda_{+}$, and $\lambda_{-}$, 
respectively. The dual subdivisions of plane tropical curves $T_{\times}, T_{+}$, and $T_{-}$defined by $\left(\widetilde{\Gamma}_{\times}, \tilde{w}_{\times}, \tilde{h}_{\times}, \widetilde{\boldsymbol{P}}_{\times}\right),\left(\widetilde{\Gamma}_{+}, \tilde{w}_{+}, \tilde{h}_{+}, \widetilde{\boldsymbol{P}}_{+}\right)$, and $\left(\widetilde{\Gamma}_{-}, \tilde{w}_{-}, \tilde{h}_{-}, \widetilde{\boldsymbol{P}}_{-}\right)$differ by fragments shown in Figure $3\left(\right.$ b). Denote by $\mathrm{W}_{\times}, \mathrm{W}_{+}$, and $\mathrm{W}_{-}$the (multiplicative) contributions of these fragments to $\mathrm{W}\left(T_{\times}\right), \mathrm{W}\left(T_{+}\right)$, and $\mathrm{W}\left(T_{-}\right)$. We have to check that $\mathrm{W}_{\times}+\mathrm{W}_{+}=\mathrm{W}_{-}$.

If either at least one of the lengths $|A B|,|B C|,|C D|,|A D|$ is even, or all the lengths $|A C|,|B D|,|F C|$ are even, then $\mathrm{W}_{\times}=\mathrm{W}_{+}=\mathrm{W}_{-}=0$. So, assume that $|A B|,|B C|,|C D|,|A D|$ are odd, and $|A C|,|B D|,|F C|$ are not all even. The first assumption yields

$$
\begin{aligned}
& \operatorname{Area}(B C F)=\operatorname{Area}(C D F)=|C F| \bmod 2, \\
& \operatorname{Area}(A B D)=\operatorname{Area}(B C D)=|B D| \bmod 2, \\
& \operatorname{Area}(A B C)=\operatorname{Area}(A C D)=|A C| \bmod 2 .
\end{aligned}
$$

Therefore, (8) and the second assumption imply that two of the lengths $|A C|,|B D|$, $|F C|$ are odd and the third one is even. Denote by $N_{\times}$(respectively, $N_{+}, N_{-}$) the total number of integer points lying in the interior of the triangles $B C F$ and $C D F$ (respectively, $A B D$ and $B C D, A B C$ and $A C D$ ), and denote by $N$ (respectively, $N^{\prime}$ ) the number of integer points lying in the interior of the quadrilateral $A B C D$ (respectively, the triangle $A B D$ ). Then $N_{+}=N-|B D|+1, N_{-}=N-|A C|+1$ and $N_{\times}=N-2 N^{\prime}-|B D|+1-|B F|-|F D|-|C F|+2$.

If $|A C|$ is even, while $|B D|$ and $|F C|$ are odd, then $\mathrm{W}_{\times}=(-1)^{N_{\times}}, \mathrm{W}_{+}=$ $(-1)^{N_{+}}$, and $\mathrm{W}_{-}=0$. Furthermore, in this case, $N_{+}=N \bmod 2$ and $N_{\times}=N+1$ mod 2, which yields $\mathrm{W}_{\times}+\mathrm{W}_{+}=0=\mathrm{W}_{-}$.

If $|B D|$ is even, while $|A C|$ and $|F C|$ are odd, then $\mathrm{W}_{\times}=(-1)^{N_{\times}}, \mathrm{W}_{+}=0$, and $\mathrm{W}_{-}=(-1)^{N_{-}}$. Furthermore, in this case, $N_{-}=N \bmod 2$ and $N_{\times}=N$ $\bmod 2$, which yields $\mathrm{W}_{\times}+\mathrm{W}_{+}=(-1)^{N}=\mathrm{W}_{-}$.

If $|F C|$ is even, while $|A C|$ and $|B D|$ are odd, then $\mathrm{W}_{\times}=0, W_{+}=(-1)^{N_{+}}$, and $\mathrm{W}_{-}=(-1)^{N_{-}}$. Furthermore, in this case, $N_{+}=N \bmod 2$ and $N_{-}=N$ $\bmod 2$, which yields $\mathrm{W}_{\times}+\mathrm{W}_{+}=(-1)^{N}=\mathrm{W}_{-}$.

\subsubsection{Degenerate case}

Lemma 20. Let $\lambda \in \Lambda_{\Delta, \alpha, \beta, g}$ be as in Section 4.6.1. Assume that some of the four lines $L_{1}, L_{2}, L_{3}$, and $L_{4}$ coincide. Then there are exactly two $\sigma$-generic combinatorial types which admit $\lambda$ as a degeneration. These combinatorial types $\lambda_{+}$and $\lambda_{-}$are perturbations of $\lambda$. Fragments of the graphs $\widetilde{\Gamma}_{+}$and $\widetilde{\Gamma}_{-}$and their images under $\tilde{h}_{+}$ and $\tilde{h}_{-}$are shown in Figure 4 (a), (b), (c) (in the cases (a), (b) and (c), the polygon corresponding to $V$ in the subdivision dual to the plane tropical curve defined by $(\Gamma, w, h)$ is a triangle, trapeze, and parallelogram, respectively). The perturbations $\lambda_{+}$, and $\lambda_{-}$are not equivalent in the same sense as in Lemma 16. Furthermore, $\operatorname{ev}\left(\mathcal{M}_{\Delta, \alpha, \beta, g}^{\lambda_{+}}\right)$and $\operatorname{ev}\left(\mathcal{M}_{\Delta, \alpha, \beta, g}^{\lambda_{-}}\right)$are on the opposite sides of $\operatorname{ev}\left(\mathcal{M}_{\Delta, \alpha, \beta, g}^{\lambda}\right)$. 
Proof. Let $(\Gamma, w, h, \boldsymbol{P})$ be a parameterized marked tropical curve of combinatorial type $\lambda$. According to Lemma 7 any connected component of $\bar{\Gamma} \backslash \boldsymbol{P}$ contains exactly one univalent vertex. Assume that the edges $E_{1}, E_{2}, E_{3}$, and $E_{4}$ are numbered in such a way that the simple path $\gamma$ in $\bar{\Gamma} \backslash \boldsymbol{P}$ connecting $V$ with a univalent vertex contains the edge $E_{4}$.

Consider a parameterized marked tropical curve $\left(\Gamma_{\circ}, w_{\circ}, h_{\circ}, \boldsymbol{P}_{\circ}\right)$, where $\Gamma_{\circ}$ is obtained from $\Gamma$ by removing the vertex $V$, the map $h_{\circ}$ is obtained by a modification of $h$ on the edges adjacent to $V$ in such a way that directions of the images of these edges do not change, $w_{\circ}$ is inherited from $\Gamma$, all the points of $\boldsymbol{P}_{\circ}$ but one are inherited from $\Gamma$, and the additional point $P_{\text {add }}$ of $\boldsymbol{P}_{\circ}$ belongs to $E_{4}$. The combinatorial type of $\left(\Gamma_{\circ}, w_{\circ}, h_{\circ}, \boldsymbol{P}_{\circ}\right)$ is $\sigma$-generic. Denote this combinatorial type by $\lambda_{\circ}$. Put $\boldsymbol{p}=h_{\circ}\left(\boldsymbol{P}_{\circ} \backslash\left\{P_{\text {add }}\right\}\right)$ and $p_{\text {add }}=h_{\circ}\left(P_{\text {add }}\right)$. According to Lemma 12, any twoparameter small perturbation $\left(\boldsymbol{p}(t), p_{\text {add }}(\tau)\right)$ of $\left(\boldsymbol{p}(0), p_{\text {add }}(0)\right)=\left(\boldsymbol{p}, p_{\text {add }}\right)$ lifts to a unique continuous family $F^{t, \tau}=\left(\Gamma_{\circ}^{t, \tau}, w_{\circ}^{t, \tau}, h_{\circ}^{t, \tau}, \boldsymbol{P}_{\circ}^{t, \tau}\right)$ in the moduli space of parameterized marked tropical curves of combinatorial type $\lambda_{\circ}$. For any fixed $t$, the one parameter family $F^{t, \cdot}$ has the following property: all the edges of $h_{\circ}^{t, \cdot}\left(\Gamma_{\circ}^{t, \cdot}\right)$ that are not contained in $\gamma^{t, \cdot}$ preserve their supporting lines.

Among the lines $L_{1}, L_{2}$, and $L_{3}$ choose a line $L_{i}$ such that the two other lines are distinct, and $L_{i}$ either coincides with one of these two lines or coincides with $L_{4}$. Change, if necessary, the numbering of lines $L_{1}, L_{2}$, and $L_{3}$ in order to have $i=3$ and the lines $L_{2}$ and $L_{3}$ non coinciding. The position of the lines $L_{1}^{t, \tau}$, $L_{2}^{t, \tau}$, and $L_{3}^{t, \tau}$ does not depend on $\tau$, and if $\boldsymbol{p}(t) \notin \operatorname{ev}\left(\mathcal{M}^{\lambda}(\Delta, \alpha, \beta, g)\right)$, then these lines do not have a common point. Thus, if the perturbation $\left(\boldsymbol{p}(t), p_{\text {add }}(\tau)\right)$ is linear, $p(t) \notin \operatorname{ev}\left(\mathcal{M}^{\lambda}(\Delta, \alpha, \beta, g)\right)$ for $t \neq 0$, and $p_{\text {add }}(\tau) \notin L_{4}$ for $\tau \neq 0$, then $L_{3}^{t, \tau}$ transports with a non-zero velocity vector relative to $L_{1}^{t, \tau} \cup L_{2}^{t, \tau}$, while $L_{4}^{t, \tau}$ (which depends only on $\tau$ ) performs another, independent, parallel transport movement with a non-zero velocity vector relative to $L_{1}^{t, \tau} \cup L_{2}^{t, \tau}$.

For a certain sign of $t$ (assume that this sign is -) and sufficiently small absolute value of $t$, the ray starting at the point $L_{2}^{t, \tau} \cap L_{3}^{t, \tau}$ and going in the direction determined by the balancing condition intersects the ray of $F^{t, \tau}=\left(\Gamma_{\circ}^{t, \tau}, w_{\circ}^{t, \tau}, h_{\circ}^{t, \tau}, \boldsymbol{P}_{\circ}^{t, \tau}\right)$ supported by $L_{1}^{t, \tau}$. Selecting $\tau=\tau(t)$ in such a way that $L_{4}^{t, \tau}$ goes through the above intersection point, gives rise to a tropical curve whose combinatorial type is a perturbation of $\lambda$.

If $L_{3}$ does not coincide with $L_{1}$, then for positive and sufficiently small values of $t$, the ray starting at the point $L_{1}^{t, \tau} \cap L_{3}^{t, \tau}$ and going in the direction determined by the balancing condition intersects the ray of $F^{t, \tau}=\left(\Gamma_{\circ}^{t, \tau}, w_{\circ}^{t, \tau}, h_{\circ}^{t, \tau}, \boldsymbol{P}_{\circ}^{t, \tau}\right)$ supported by $L_{2}^{t, \tau}$, and a construction as above gives rise to a tropical curve whose combinatorial type is a perturbation of $\lambda$. If $L_{3}$ coincides with $L_{1}$, then for positive and sufficiently small values of $t$, the ray starting at the point $L_{1}^{t, \tau} \cap L_{2}^{t, \tau}$ and going in the direction determined by the balancing condition intersects the ray of $F^{t, \tau}=\left(\Gamma_{\circ}^{t, \tau}, w_{\circ}^{t, \tau}, h_{\circ}^{t, \tau}, \boldsymbol{P}_{\circ}^{t, \tau}\right)$ supported by $L_{3}^{t, \tau}$, and again a construction as above 
gives rise to a tropical curve whose combinatorial type is a perturbation of $\lambda$.

Since some of the lines $L_{1}, L_{2}, L_{3}$, and $L_{4}$ coincide, there are at most two combinatorial types that can be perturbations of $\lambda$. On the other hand, we constructed two distinct perturbations of $\lambda$ (denote the first one by $\lambda_{-}$and the second one by $\lambda_{+}$). Moreover, the images $\operatorname{ev}\left(\mathcal{M}_{\Delta, \alpha, \beta, g}^{\lambda_{+}}\right)$and $\operatorname{ev}\left(\mathcal{M}_{\Delta, \alpha, \beta, g}^{\lambda_{-}}\right)$are on the opposite sides of $\operatorname{ev}\left(\mathcal{M}_{\Delta, \alpha, \beta, g}^{\lambda}\right)$.

Lemma 21. Let $\lambda, \lambda_{+}$, and $\lambda_{-}$be as in Lemma 20. Then $\mathrm{W}\left(\lambda_{+}\right)=\mathrm{W}\left(\lambda_{-}\right)$.

Proof. Consider parameterized marked tropical curves $\left(\widetilde{\Gamma}_{+}, \tilde{w}_{+}, \tilde{h}_{+}, \widetilde{\boldsymbol{P}}_{+}\right)$and $\left(\widetilde{\Gamma}_{-}, \tilde{w}_{-}, \tilde{h}_{-}, \widetilde{\boldsymbol{P}}_{-}\right)$of combinatorial types $\lambda_{+}$and $\lambda_{-}$. The dual subdivisions of plane tropical curves $T_{+}$and $T_{-}$defined by $\left(\widetilde{\Gamma}_{+}, \tilde{w}_{+}, \tilde{h}_{+}, \widetilde{\boldsymbol{P}}_{+}\right)$and $\left(\widetilde{\Gamma}_{-}, \tilde{w}_{-}, \tilde{h}_{-}, \widetilde{\boldsymbol{P}}_{-}\right)$differ by fragments shown in Figure 4 (d), 4 (e), or 4 (f). These fragments correspond to two splittings of the polygon dual to $h(V)$, and their (multiplicative) contributions $\mathrm{W}_{+}$and $\mathrm{W}_{-}$to $\mathrm{W}\left(T_{+}\right)$and $\mathrm{W}\left(T_{-}\right)$, respectively, are equal in each of the cases $4(\mathrm{~d})$, (e), (f). Indeed, $\mathrm{W}_{+}$and $\mathrm{W}_{-}$both vanish if

- in the case $4(\mathrm{~d})$, at least one of the lengths $|A B|,|A C|,\left|B D_{1}\right|=\left|C D_{2}\right|$, $\left|C D_{1}\right|=\left|B D_{2}\right|$ in Figure $4(\mathrm{~d})$ is even,

- in the cases 4 (e), (f), at least one of the lengths $|A B|,|B C|,|C D|,|A D|$ is even.

If the aforementioned lengths are odd then, in the case 4 (d) one has $\left|A D_{1}\right|=\left|A D_{2}\right|$ $\bmod 2$, and in the cases 4 (e) and 4(f), one has $|A C|=|B D| \bmod 2$. This yields $\mathrm{W}_{+}=\mathrm{W}_{-}$.

4.7. Tropically generic configurations. A configuration $\boldsymbol{p} \in \Omega(\Delta, \alpha, \beta, g)$ is called $(\Delta, \alpha, \beta, g)$-generic (resp., almost $(\Delta, \alpha, \beta, g)$-generic) if the inverse image $(\mathrm{ev})^{-1}(\boldsymbol{p})$ of $\boldsymbol{p}$ under the evaluation map ev: $\mathcal{M}_{\Delta, \alpha, \beta, g} \rightarrow \Omega(\Delta, \alpha, \beta, g)$ consists of parameterized marked tropical curves of $\sigma$-generic (resp., of $\sigma$-generic or injective codimension 1) combinatorial types.

We say that a parameterized plane tropical curve $(\Gamma, w, h)$ of degree $\Delta^{\alpha, \beta}$ and genus $g$ matches a configuration $\boldsymbol{p}=\boldsymbol{p}^{b} \cup \boldsymbol{p}^{\sharp} \in \Omega(\Delta, \alpha, \beta, g)$ if $\boldsymbol{p} \subset h(\Gamma)$, and any point $p_{k} \in \boldsymbol{p}^{\mathrm{b}}$ is contained in the image of a left end of weight $2 i_{k}-1$, where the positive integer $i_{k}$ is determined by the inequalities $\sum_{j<i_{k}} \alpha_{j}<k \leq \sum_{j \leq i_{k}} \alpha_{j}$. A $(\Delta, \alpha, \beta, g)$-generic configuration $\boldsymbol{p}=\boldsymbol{p}^{\mathrm{b}} \cup \boldsymbol{p}^{\sharp} \in \Omega(\Delta, \alpha, \beta, g)$ is called tropically generic if any parameterized plane tropical curve matching $\boldsymbol{p}$ and having the degree $\Delta^{\alpha, \beta}$ and the genus $g$ defines a plane tropical curve $T$ which satisfies the following properties: $T$ is nodal, and no point in $\boldsymbol{p}^{\sharp}$ coincides with a vertex of $T$.

Lemma 22. The complement in $\Omega(\Delta, \alpha, \beta, g)$ of the subset formed by the tropically generic configurations is a finite closed polyhedral complex of positive codimension. 

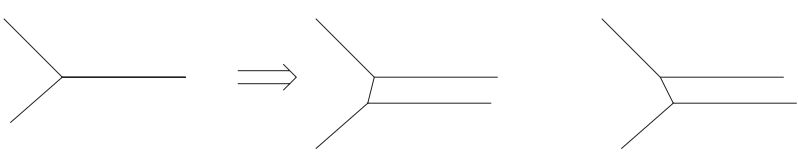

(a)
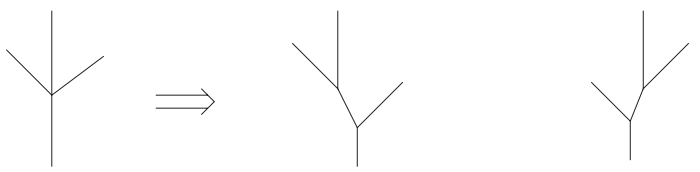

(b)
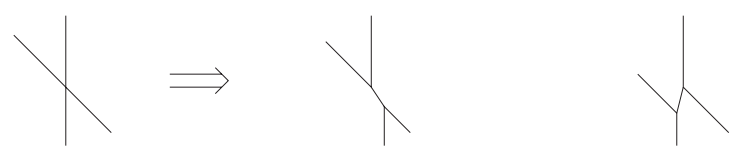

(c)
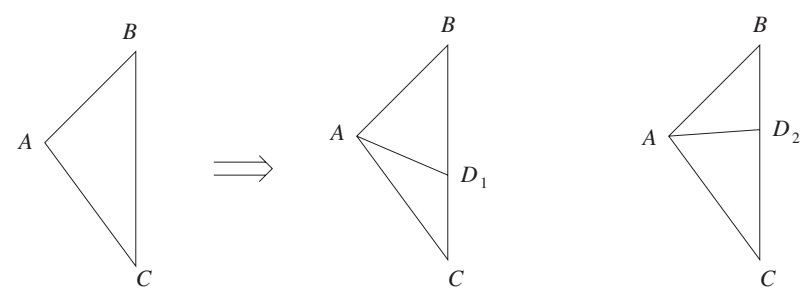

(d)
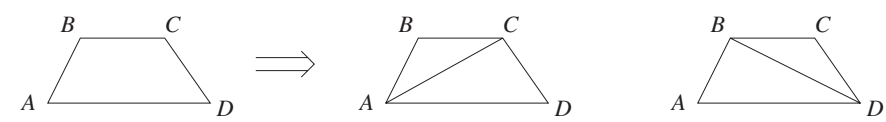

(e)
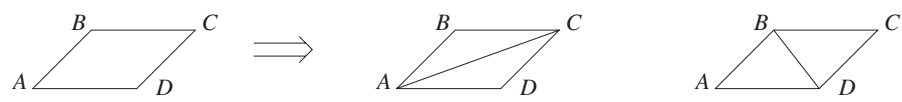

(f)

Figure 4. Degenerate second bifurcations. 
Proof. The subset formed by the non-tropically generic configurations in the space $\Omega(\Delta, \alpha, \beta, g)$ is a projection of $\cup_{\lambda \in \Lambda_{\Delta, \alpha, \beta, g}} \mathcal{B}^{\lambda}$, where $\mathscr{B}^{\lambda} \subset \mathcal{P}^{\lambda}$ is described by a disjunction of a finite collection of systems of linear equations and linear inequalities in graphic coordinates. Since the set $\Lambda_{\Delta, \alpha, \beta, g}$ is finite, it remains to prove that the tropically generic configurations form an open dense subset in $\Omega(\Delta, \alpha, \beta, g)$.

As it immediately follows from Lemma 11 (and finiteness of $\Lambda_{\Delta, \alpha, \beta, g}$ ), the $(\Delta, \alpha, \beta, g)$-generic configurations form an open dense subset in $\Omega(\Delta, \alpha, \beta, g)$. Denote by $O$ the set of the $(\Delta, \alpha, \beta, g)$-generic configurations $p \in \Omega(\Delta, \alpha, \beta, g)$ such that any parameterized plane tropical curve which matches $p$ and has the degree $\Delta^{\alpha, \beta}$ and the genus $g$ is simply parameterized. Since for any parameterized plane tropical curve one can always choose a configuration $\boldsymbol{P} \subset \Gamma$ of marked points to obtain a parameterized marked tropical curve $(\Gamma, w, h, \boldsymbol{P})$, Lemma 9 implies that $O$ is an open dense subset in $\Omega(\Delta, \alpha, \beta, g)$.

Denote by $C_{1}$ the set of the configurations $\boldsymbol{p}=\boldsymbol{p}^{\mathrm{b}} \cup \boldsymbol{p}^{\sharp} \in O$ admitting a simply parameterized plane tropical curve $(\Gamma, w, h)$ which matches $p$, has the degree $\Delta^{\alpha, \beta}$ and the genus $g$, and satisfies the following property: the plane tropical curve defined by $(\Gamma, w, h)$ has a vertex at one of the points of $\boldsymbol{p}^{\sharp}$. Clearly, $C_{1}$ is a closed nowhere dense subset of $O$.

Denote by $C_{2}$ the set of the configurations $p \in O$ admitting a simply parameterized plane tropical curve $(\Gamma, w, h)$ which matches $\boldsymbol{p}$, has the degree $\Delta^{\alpha, \beta}$ and the genus $g$, and satisfies the following property: the plane tropical curve defined by $(\Gamma, w, h)$ is not nodal. For a combinatorial type $\lambda \in \Lambda(\Delta, \alpha, \beta, g)$ of simply parameterized marked tropical curves, consider the image in $Q^{\lambda}$ of the points of $\mathcal{P}^{\lambda}$ corresponding to parameterized marked tropical curves defining non-nodal plane tropical curves. This image is a closed nowhere dense subset of $Q^{\lambda}$ as can be shown using the same arguments as in the proof of [7], Proposition 2.23. Hence, $C_{2}$ is a closed nowhere dense subset of $O$. Thus, the tropically generic configurations form an open dense subset in $\Omega(\Delta, \alpha, \beta, g)$.

Lemma 23. Let $\boldsymbol{p} \in \Omega(\Delta, \alpha, \beta, g)$ be a tropically generic configuration. Then any curve in $\mathcal{T}^{\mathrm{irr}}(\Delta, \alpha, \beta, g, \boldsymbol{p})$ can be parameterized by an element of the inverse image $(\mathrm{ev})^{-1}(\boldsymbol{p})$ of $\boldsymbol{p}$ under the evaluation map $\mathcal{M}_{\Delta, \alpha, \beta, g} \rightarrow \Omega(\Delta, \alpha, \beta, g)$.

Proof. Pick an element $T$ in $\mathcal{T}^{\text {irr }}(\Delta, \alpha, \beta, g, \boldsymbol{p})$, and consider a simple parameterization $(\Gamma, w, h)$ of $T$. The configuration $\boldsymbol{p}$ lifts to a configuration $\boldsymbol{P} \subset \Gamma$. Assume that $\bar{\Gamma} \backslash \boldsymbol{P}$ has a component containing either a loop, or two univalent vertices. Then there exists a one-dimensional family of simply parameterized plane tropical curves $\left(\Gamma, w, h_{t}\right)$ such that $h_{t}(\boldsymbol{P})=\boldsymbol{p}$ for any $t$, and the coordinates of the images of vertices of $\Gamma$ depend linearly on $t$. Hence, this family degenerates either to a situation of collision of two vertices of $\Gamma$, or to a situation of collision of a point in $\boldsymbol{P}$ and a vertex of $\Gamma$. The both cases contradict the fact that $\boldsymbol{p}$ is tropically generic. 
Lemma 24. For any tropically generic configuration $p \in \Omega(\Delta, \alpha, \beta, g)$, the set $\mathcal{T}^{\mathrm{irr}}(\Delta, \alpha, \beta, g, \boldsymbol{p})$ is finite.

Proof. The lemma follows from Lemma 12, Lemma 23, and finiteness of the set $\Lambda_{\Delta, \alpha, \beta, g}$.

4.8. Multi-tropically generic configurations. Let $\delta_{*}$ be the set of the 4-tuples $\left(\Delta_{*}, \alpha_{*}, \beta_{*}, g_{*}\right)$ formed by a left-nondegenerate convex lattice polygon $\Delta_{*}$, elements $\alpha_{*}$ and $\beta_{*}$ in $\mathcal{C}$ such that $J \alpha_{*}+J \beta_{*}=\left|\sigma_{*}\right|$ (where $\sigma_{*}$ is the intersection of $\Delta_{*}$ with its left vertical supporting line), and an integer $g_{*}$. Define an addition operation in $\delta_{*}$ in the same way as in $\delta$ (see Section 3.3).

A partition $\bigsqcup_{j=1}^{\ell} \boldsymbol{p}^{(j)}$ of $\boldsymbol{p} \in \Omega(\Delta, \alpha, \beta, g)$ is called compatible with a splitting

$$
(\Delta, \alpha, \beta, g)=\sum_{j=1}^{\ell}\left(\Delta^{(j)}, \alpha^{(j)}, \beta^{(j)}, g^{(j)}\right)
$$

in $\delta_{*}$ if $\boldsymbol{p}^{(j)} \in \Omega\left(\Delta^{(j)}, \alpha^{(j)}, \beta^{(j)}, g^{(j)}\right)$ for any $j=1, \ldots, \ell$. A configuration $p \in \Omega(\Delta, \alpha, \beta, g)$ is called multi-tropically generic if for any splitting

$$
(\Delta, \alpha, \beta, g)=\sum_{j=1}^{\ell}\left(\Delta^{(j)}, \alpha^{(j)}, \beta^{(j)}, g^{(j)}\right)
$$

in $\delta_{*}$ and any partition $\boldsymbol{p}=\bigsqcup_{j=1}^{\ell} \boldsymbol{p}^{(j)}$ compatible with this splitting the following holds:

- each configuration $\boldsymbol{p}^{(j)}$ is tropically generic,

- any sum $\sum_{j=1}^{\ell} T^{(j)}$ is a nodal plane tropical curve whenever $T^{(j)}$ is a plane tropical curve defined by a parameterized tropical curve which belongs to the inverse image $(\mathrm{ev})^{-1}\left(\boldsymbol{p}^{(j)}\right)$ of $\boldsymbol{p}^{(j)}$ under the evaluation map ev: $\mathcal{M}_{\Delta^{(j)}, \boldsymbol{\alpha}^{(j)}, \beta^{(j)}, g^{(j)}} \rightarrow$ $\Omega\left(\Delta^{(j)}, \alpha^{(j)}, \beta^{(j)}, g^{(j)}\right)$.

Lemma 25. The complement in $\Omega(\Delta, \alpha, \beta, g)$ of the subset formed by the multitropically generic configurations is a finite closed polyhedral complex of positive codimension.

Proof. Consider the subset $U \subset \Omega(\Delta, \alpha, \beta, g)$ formed by the configurations $\boldsymbol{p}$ such that, for any splitting $(\Delta, \alpha, \beta, g)=\sum_{j=1}^{\ell}\left(\Delta^{(j)}, \alpha^{(j)}, \beta^{(j)}, g^{(j)}\right)$ in $\delta_{*}$ and any partition $\boldsymbol{p}=\bigsqcup_{j=1}^{\ell} \boldsymbol{p}^{(j)}$ compatible with the given splitting, all the configurations $\boldsymbol{p}^{(1)}, \ldots, \boldsymbol{p}^{(\ell)}$ are tropically generic. Lemma 22 implies that $U$ is an open dense subset of $\Omega(\Delta, \alpha, \beta, g)$. Now the statement of the lemma follows from the fact that the sum of several nodal plane tropical curves can be always made nodal by arbitrarily small parallel shifts of summands. 
Lemma 26. For any multi-tropically generic configuration $\boldsymbol{p} \in \Omega(\Delta, \alpha, \beta, g)$, the set $\mathcal{T}(\Delta, \alpha, \beta, g, \boldsymbol{p})$ is finite.

Proof. The statement is an immediate consequence of Lemma 24.

Lemma 27. Let $\boldsymbol{p} \in \Omega(\Delta, \alpha, \beta, g)$ be a multi-tropically generic configuration. Then

$$
\mathrm{W}(\Delta, \alpha, \beta, g, \boldsymbol{p})=\sum \prod_{j=1}^{\ell} \mathrm{W}^{\mathrm{irr}}\left(\Delta^{(j)}, \alpha^{(j)}, \beta^{(j)}, g^{(j)}, \boldsymbol{p}^{(j)}\right),
$$

where the sum is taken over all unordered splittings

$$
(\Delta, \alpha, \beta, g)=\sum_{j=1}^{\ell}\left(\Delta^{(j)}, \alpha^{(j)}, \beta^{(j)}, g^{(j)}\right)
$$

in $\gamma_{*}$ and all compatible partitions of $\boldsymbol{p}$.

Proof. Straightforward.

4.9. Proof of Theorem 1. Notice that it is enough to establish the invariance of the numbers $\mathrm{W}^{\text {irr }}(\Delta, \alpha, \beta, g)$, since the invariance of $\mathrm{W}(\Delta, \alpha, \beta, g)$ will then follow from (9).

Pick two tropically generic configurations $\boldsymbol{p}$ and $\boldsymbol{q}$ in $\left(L_{-\infty}\right)^{\|\alpha\|} \times\left(\mathbb{R}^{2}\right)^{r-\|\alpha\|}$, and connect them by a path $\xi \subset\left(L_{-\infty}\right)^{\|\alpha\|} \times\left(\mathbb{R}^{2}\right)^{r-\|\alpha\|}$ such that

- $\xi$ consists of $(\Delta, \alpha, \beta, g)$-generic configurations and finitely many almost $(\Delta, \alpha, \beta, g)$-generic configurations,

- for any almost $(\Delta, \alpha, \beta, g)$-generic configuration $z \in \xi$ and any injective codimension 1 combinatorial type of parameterized marked tropical curves in $(\mathrm{ev})^{-1}(z) \subset \mathcal{M}_{\Delta, \alpha, \beta, g}$, the path $\xi$ intersects $\operatorname{ev}\left(\mathcal{M}_{\Delta, \alpha, \beta, g}^{\lambda}\right)$ transversally.

According to Lemmas $15,17,19$, and 21 , the value of $\sum_{\lambda \in \mathrm{ev}^{-1}(z)} \mathrm{W}(\lambda)$ is the same for all $(\Delta, \alpha, \beta, g)$-generic configurations $z \in \xi$. In particular, $\mathrm{W}^{\text {irr }}(\Delta, \alpha, \beta, g, \boldsymbol{p})=$ $\mathrm{W}^{\mathrm{irr}}(\Delta, \alpha, \beta, g, \boldsymbol{q})$.

\section{Proof of the recursive formulas}

\subsection{Auxiliary lemmas}

Lemma 28 (cf. [3], proof of Theorem 4.3). Let $\Delta, \alpha, \beta$, and $g$ be as in Theorem 3. Fix positive real numbers $\varepsilon$ and $N$, and consider a multi-tropically generic configuration $\boldsymbol{p}=\left(\boldsymbol{p}^{b}, \boldsymbol{p}^{\sharp}\right) \in \Omega(\Delta, \alpha, \beta, g)$ such that 
- the second coordinates of all the points in p belong to the interval $(-\varepsilon, \varepsilon)$,

- the first coordinate of one point in $\boldsymbol{p}^{\sharp}$ is smaller than $-N$, while the first coordinates of all other points in $\boldsymbol{p}^{\sharp}$ belong to the interval $(-\varepsilon, \varepsilon)$.

Then for any tropical curve $T \in \mathcal{T}(\Delta, \alpha, \beta, g, p)$, the second coordinates of all trivalent vertices of $T$ belong to the interval $(-\varepsilon, \varepsilon)$. Furthermore, if $N$ is sufficiently large with respect to $\varepsilon$, there exist real numbers $a$ and $b$ satisfying the inequalities $-N<a<b<-\varepsilon$ and satisfying the following condition: for any tropical curve $T \in \mathcal{T}(\Delta, \alpha, \beta, g, \boldsymbol{p})$ the intersection of $T$ with the rectangle $\left\{(x, y) \in \mathbb{R}^{2}: a \leq\right.$ $x \leq b$ and $-\varepsilon \leq y \leq \varepsilon\}$ does not contain vertices of $T$ and consists of horizontal segments.

Proof. Consider a tropical curve $T \in \mathcal{T}(\Delta, \alpha, \beta, g, \boldsymbol{p})$. Among the trivalent vertices of $T$ choose a vertex $v=\left(v_{1}, v_{2}\right)$ having the maximal second coordinate. The curve $T$ has an end starting at $v$ and pointing upwards. This end is orthogonal to one of the upper sides of $\Delta$, and thus, is of weight 1 and of direction either $(0,1)$ or $(1,1)$. Hence, $T$ should have another edge which starts at $v$ and does not point downwards. Consider a simple parameterization $(\Gamma, w, h)$ of the irreducible subcurve $T^{0}$ of $T$ such that $v \in T^{0}$, and denote by $\boldsymbol{P}$ the lifting of $\boldsymbol{p}$ to $\Gamma$. If $v_{2}>\varepsilon$, the connected component of $\bar{\Gamma} \backslash \boldsymbol{P}$ containing $h^{-1}(v)$ has at least two ends, and thus, $(\Gamma, w, h, \boldsymbol{P})$ is not a parameterized marked tropical curve. This contradicts Lemma 23. In the same way one can show that the curve $T$ does not have vertices below the line $y=-\varepsilon$. This proves the first statement of the lemma.

Denote by $R$ the rectangle $\left\{(x, y) \in \mathbb{R}^{2}:-N \leq x \leq-\varepsilon\right.$ and $\left.-\varepsilon \leq y \leq \varepsilon\right\}$. Let $T^{1}$ be an irreducible subcurve of $T$ such that the intersection of $T^{1}$ with the interior of $R$ is non-empty, and let $\left(\Gamma^{1}, w^{1}, h^{1}\right)$ be a simple parameterization of $T^{1}$. As follows from Lemma 23 and the first statement of the current lemma, the image under $h$ of any path $\gamma \subset \bar{\Gamma} \backslash \boldsymbol{P}$ does not intersect one of the two horizontal edges of $R$. Thus, for any point $\left(x_{1}, y_{1}\right)$ belonging to the interior of $R$ and to a non-horizontal edge of $T^{1}$, there exists a path $\gamma \subset \bar{\Gamma} \backslash \boldsymbol{P}$ such that $h(\gamma)$ contains $\left(x_{1}, y_{1}\right)$ and is the graph of a strictly monotone function $f$ defined either on the interval $\left[-N, x_{1}\right]$, or on the interval $\left[x_{1}-\varepsilon\right]$. Since there are only finitely many slopes that can be realized by the edges of a tropical curve with Newton polygon $\Delta$, the length of the definition interval of $f$ is bounded from above by a constant depending only on $\Delta$. This proves the second statement of the lemma.

Lemma 29. Consider a non-degenerate lattice polygon $\delta$ whose projection to the horizontal coordinate axis coincides with the segment $[0,1]$. Put $\sigma_{1}=\delta \cap\{x=0\}$, $\sigma_{2}=\delta \cap\{x=1\}$, and introduce the vectors $u_{1}=(-1,0), u_{2}=(1,0)$. Fix a point $p \in \mathbb{R}^{2} \subset \widehat{\mathbb{R}}^{2}$ and an ordered splitting $\left|\sigma_{1}\right|=n_{1,1}+\cdots+n_{1, m_{1}}$ (respectively, $\left|\sigma_{2}\right|=n_{2,1}+\cdots+n_{2, m_{1}}$ ) of $\left|\sigma_{1}\right|$ (respectively, of $\left|\sigma_{2}\right|$ ) into positive integer summands. Fix also two non-increasing sequences of real numbers $y_{1,1}, \ldots, y_{1, m_{1}}$ 
and $y_{2,1}, \ldots, y_{2, m_{2}}$. (In the case $\left|\sigma_{i}\right|=0$, the sequences $n_{i, 1}, \ldots, n_{i, m_{i}}$ and $y_{i, 1}, \ldots, y_{i, m_{i}}$ are empty.) Then there exists a parameterized plane tropical curve $(\Gamma, w, h)$ satisfying the following conditions:

(1) $\Gamma$ has genus 0 and degree $\left(n_{1,1} u_{1}, \ldots, n_{1, m_{1}} u_{1}, n_{2,1} u_{2}, \ldots, n_{2, m_{2}} u_{2}, u_{3}, u_{4}\right)$, where $u_{3}$ and $u_{4}$ are primitive integer outward normal vectors of the two nonvertical sides of $\delta$,

(2) $\Gamma$ has $m_{1}$ ends $E_{1,1}, \ldots, E_{1, m_{1}}$ such that, for any $k=1, \ldots, m_{1}$, the end $E_{1, k}$ is of weight $n_{1, k}$, and $h\left(E_{1, k}\right)$ is a horizontal negatively directed ray which is contained in the line $y=y_{1, k}$,

(3) $\Gamma$ has $m_{2}$ ends $E_{2,1}, \ldots, E_{2, m_{2}}$ such that, for any $k=1, \ldots, m_{2}$, the end $E_{2, k}$ is of weight $n_{2, k}$, and $h\left(E_{2, k}\right)$ is a horizontal positively directed ray which is contained in the line $y=y_{2, k}$,

(4) $p \in h(\Gamma)$.

Furthermore, if all the numbers $y_{1,1}, \ldots, y_{1, m_{1}}, y_{2,1}, \ldots, y_{2, m_{2}}$ differ from the second coordinate of $p$, then all parameterized plane tropical curves having the above properties define the same plane tropical curve, and p belongs to the interior of a non-horizontal edge of this curve.
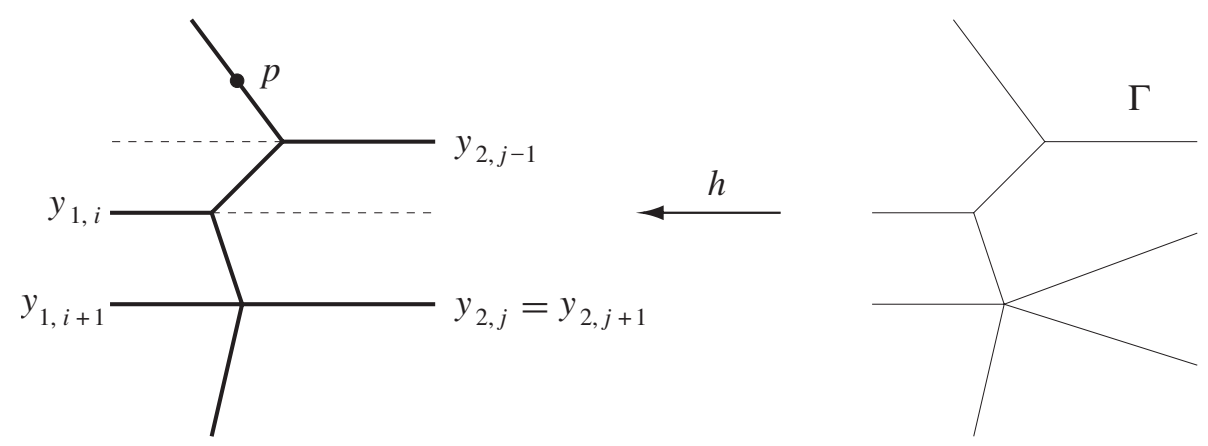

Figure 5. Curve $(\Gamma, w, h)$ in Lemma 29.

Proof. Any plane tropical curve having a parameterization with the described properties can be constructed in the following way. Take the union $\beth$ of all the lines $y=y_{1,1}, \ldots, y=y_{1, m_{1}}, y=y_{2,1}, \ldots, y=y_{2, m_{2}}$, and consider a broken line $\mathscr{L}$ such that

- any vertex of $\mathscr{L}$ belongs to $\beth$,

- any edge of $\mathscr{L}$ has a rational nonzero slope,

- the two unbounded edges of $\mathscr{L}$ have directions determined by the vectors $u_{3}$ and $u_{4}$, 
- for any vertex $v$ of $\mathscr{L}$, the two primitive integer vectors $e_{1}$ and $e_{2}$ starting at $v$ and directed along the adjacent edges of $\mathscr{L}$ satisfy the relation

$$
e_{1}+e_{2}+\sum_{y_{1, k}=v_{y}} n_{1, k} u_{1}+\sum_{y_{2, k}=v_{y}} n_{2, k} u_{2}=0
$$

where $y=v_{y}$ is the line containing $v$.

Make a horizontal shift of $\mathscr{L}$ in order to obtain a broken line containing $p$, and extend the result (in a unique possible way) to a plane tropical curve which admits a parameterization satisfying the properties (1)-(3). This proves the existence. The second statement of the lemma immediately follows from the construction.

Consider the subset $\mathcal{X} \subset \Omega(\Delta, \alpha, \beta, g)$ formed by the multi-tropically generic configurations $\boldsymbol{p}=\boldsymbol{p}^{\mathrm{b}} \cup \boldsymbol{p}^{\sharp}$ satisfying the following property: for any point $p \in \boldsymbol{p}^{\sharp}$, there exists a number $M$ such that any configuration $\tilde{p} \in \Omega(\Delta, \alpha, \beta, g)$ obtained from $p$ by a horizontal shift of $p$ to a point whose first coordinate is smaller than $M$ and different from $-\infty$ is multi-tropically generic.

Lemma 30. The subset $\mathcal{X}$ is open dense in $\Omega(\Delta, \alpha, \beta, g)$.

Proof. Straightforward from Lemma 25.

Lemma 31. Let $\Delta, \alpha, \beta$, and $g$ be as in Theorem 3. Choose $T, \alpha^{\prime}, \beta^{\prime}$, and $g^{\prime}$ satisfying the conditions (3), a configuration $\boldsymbol{p}=\boldsymbol{p}^{b} \cup \boldsymbol{p}^{\sharp} \in \mathcal{X}$, and a point $p \in \boldsymbol{p}^{\sharp}$. Then any subconfiguration $\boldsymbol{q} \subset \boldsymbol{p}$ such that $\boldsymbol{q} \in \Omega\left(l_{\top}(\Delta), \alpha^{\prime}, \beta^{\prime}, g^{\prime}\right)$ and $\boldsymbol{q}^{\sharp}=\boldsymbol{p}^{\sharp} \backslash\{p\}$ is multi-tropically generic.

Proof. Consider a splitting $\left(l_{\top}(\Delta), \alpha^{\prime}, \beta^{\prime}, g^{\prime}\right)=\sum_{i=1}^{m}\left(\Delta^{(i)}, \alpha^{(i)}, \beta^{(i)}, g^{(i)}\right)$ in $\delta_{*}$, and a partition $\bigsqcup_{i=1}^{m} \boldsymbol{q}^{(i)}$ of $\boldsymbol{q}$ compatible with this splitting. All the polygons $\Delta^{(1)}, \ldots, \Delta^{(m)}$ are in $\Xi$ (it follows from Remark 1 and the fact that $l_{\top}(\Delta) \in \Xi$ ). For each $i=1, \ldots, m$, pick a parameterized plane tropical curve $\left(\Gamma^{(i)}, w^{(i)}, h^{(i)}\right)$ matching $\boldsymbol{q}^{(i)}$ and having the degree $\left(\Delta^{(i)}\right)^{\alpha^{(i)}, \beta^{(i)}}$ and the genus $g^{(i)}$.

We say that a left end of $\Gamma^{(i)}$ is marked if this end terminates at a marked univalent vertex of $\Gamma^{(i)}$. Among the non-marked left ends of $\coprod_{i=1}^{m} \Gamma^{(i)}$ choose $\left\|\beta^{\prime}-\beta\right\|$ ends whose weights fit the sequence $\beta^{\prime}-\beta$, and denote the chosen set by $\mathcal{E}$. Consider a polygon $\delta$ such that

- the projection of $\delta$ to the horizontal axis coincides with the segment $[0,1]$,

- the sides $\sigma_{1}=\delta \cap\{x=0\}$ and $\sigma_{2}=\delta \cap\{x=1\}$ satisfy $\left|\sigma_{1}\right|=J(\alpha)-J\left(\alpha^{\prime}\right)$, $\left|\sigma_{2}\right|=J\left(\beta^{\prime}\right)-J(\beta)$,

- the two non-vertical sides of $\delta$ are respectively parallel to the sides $T(\Delta, T)$ and $\perp(\Delta, T)$ of $\Delta$ which are defined as follows: the side $T(\Delta, T)$ is of slope -1 and 
not a neighbor of $\sigma$ if $\overrightarrow{0} \in T$, and $T(\Delta, T)$ is the non-vertical side adjacent to the upper vertex of $\sigma$ otherwise; the side $\perp(\Delta, T)$ is of slope 0 and not a neighbor of $\sigma$ if $\overrightarrow{-1} \in \top$, and $\perp(\Delta, T)$ is the non-vertical side adjacent to the lower vertex of $\sigma$ otherwise.

The conditions (3) imply that $\delta$ is nondegenerate. Put $m_{1}=\left\|\alpha-\alpha^{\prime}\right\|$, consider a nonincreasing sequence $y_{1,1}, \ldots, y_{1, m_{1}}$ of second coordinates of points in $\boldsymbol{p}^{\mathrm{b}} \backslash \boldsymbol{q}^{\mathrm{b}}$, and denote by $n_{1,1}, \ldots, n_{1, m_{1}}$ the weights prescribed to the points of $\boldsymbol{p}^{\mathrm{b}} \backslash \boldsymbol{q}^{\mathrm{b}}$ by the sequence $\alpha$. Furthermore, put $m_{2}=\left\|\beta^{\prime}-\beta\right\|$, consider a non-increasing sequence $y_{2,1}, \ldots, y_{2, m_{2}}$ of second coordinates of the images of terminal univalent vertices of left ends belonging to $\mathcal{E}$, and denote by $n_{2,1}, \ldots, n_{2, m_{2}}$ the weights of the corresponding left ends. Take a parameterized plane tropical curve $(\Gamma, w, h)$ satisfying the conditions (1)-(4) of Lemma 29. Due to a possibility to make a negative horizontal shift of $p$ and simultaneously compose $h$ with this shift (see the definition of $\mathcal{X}$ ), we can assume that there exists a vertical line $x=c$ such that the images of vertices of $\Gamma$ lie in the left half-plane delimited by this line, and the images of non-univalent vertices of $\bigsqcup_{i} \Gamma^{(i)}$ lie in the right half-plane. In particular, the line $x=c$ crosses the images of all left ends belonging to $\mathcal{E}$ and the horizontal positively directed images of ends of $\Gamma$.

Cut along the preimages of $x=c$ the left ends belonging to $\mathcal{E}$ and the ends of $\Gamma$ whose images are horizontal and positively directed, and remove the trivial pieces of the edges cut. The natural gluing of remaining pieces of $\Gamma$ and $\bigsqcup_{i=1}^{m} \Gamma^{(i)}$ gives rise to a collection of parameterized plane tropical curves $\left(\widetilde{\Gamma}^{(j)}, \tilde{w}^{(j)}, \tilde{h}^{(j)}\right)$, $j=1, \ldots, \ell$, a splitting $(\Delta, \alpha, \beta, g)=\sum_{j=1}^{\ell}\left(\tilde{\Delta}^{(j)}, \tilde{\alpha}^{(j)}, \tilde{\beta}^{(j)}, \tilde{g}^{(j)}\right)$ in $\delta$, and a partition $\bigsqcup_{j=1}^{\ell} \tilde{\boldsymbol{p}}^{(j)}$ of $\boldsymbol{p}$ which satisfy the following properties:

- each curve $\left(\tilde{\Gamma}^{(j)}, \tilde{w}^{(j)}, \tilde{h}^{(j)}\right)$ matches the configuration $\tilde{\boldsymbol{p}}^{(j)}$ and has the degree $\left(\tilde{\Delta}^{(j)}\right)^{\tilde{\alpha}^{(j)}, \tilde{\beta}^{(j)}}$ and the genus $\tilde{g}^{(j)}$,

- the partition $\bigsqcup_{j=1}^{\ell} \tilde{\boldsymbol{p}}^{(j)}$ is compatible with the splitting

$$
(\Delta, \alpha, \beta, g)=\sum_{j=1}^{\ell}\left(\tilde{\Delta}^{(j)}, \tilde{\alpha}^{(j)}, \tilde{\beta}^{(j)}, \tilde{g}^{(j)}\right) .
$$

Since the configuration $\boldsymbol{p}$ is multi-tropically generic, each configuration $\tilde{\boldsymbol{p}}^{(j)}, j=$ $1, \ldots, \ell$ is tropically generic. This implies, that for any $j=1, \ldots, \ell$ the curve $\left(\widetilde{\Gamma}^{(j)}, \tilde{w}^{(j)}, \tilde{h}^{(j)}\right)$ defines a nodal plane tropical curve $\widetilde{T}^{(j)}$ such that no point in $\left(\tilde{\boldsymbol{p}}^{(j)}\right)^{\#}$ is a vertex of $\widetilde{T}^{(j)}$. Moreover, according to Lemma 23 , the lift $\widetilde{\boldsymbol{P}}^{(j)}$ of $\tilde{\boldsymbol{p}}^{(j)}$ to $\widetilde{\Gamma}^{(j)}$ produces a parameterized marked tropical curve $\left(\tilde{\Gamma}^{(j)}, \tilde{w}^{(j)}, \tilde{h}^{(j)}, \widetilde{\boldsymbol{P}}^{(j)}\right)$, and thus, the sum $\widetilde{T}^{(1)}+\cdots+\widetilde{T}^{(\ell)}$ is a nodal plane tropical curve. Hence, the configuration $\boldsymbol{q}$ is multi-tropically generic. 
5.2. Proof of Theorem 3. Choose positive real numbers $\varepsilon$ and $N$ satisfying the inequality $\varepsilon<N$, and consider a configuration $\boldsymbol{p}=\left(\boldsymbol{p}^{\mathrm{b}}, \boldsymbol{p}^{\sharp}\right) \in \mathcal{X} \subset \Omega(\Delta, \alpha, \beta, g)$ such that the second coordinates of all the points in $p$ belong to the interval $(-\varepsilon, \varepsilon)$, the first coordinate of one point $p \in \boldsymbol{p}^{\#}$ is smaller than $-N$, and the first coordinates of the other points in $\boldsymbol{p}^{\sharp}$ belong to the interval $(-\varepsilon, \varepsilon)$. Assume that the numbers $\varepsilon$ and $N$ are chosen in such a way that, for some numbers $a$ and $b$ satisfying the inequalities $-N<a<b<-\varepsilon$, the intersection of any tropical curve in $\mathcal{T}(\Delta, \alpha, \beta, g, \boldsymbol{p})$ with the rectangle $R_{a}^{b}=\left\{(x, y) \in \mathbb{R}^{2}: a \leq x \leq b\right.$ and $\left.-\varepsilon \leq y \leq \varepsilon\right\}$ consists of horizontal segments (the existence of such $\varepsilon$ and $N$ is guaranteed by Lemma 28). Consider a tropical curve $T \in \mathcal{T}(\Delta, \alpha, \beta, g, \boldsymbol{p})$ without edges of even weight.

Suppose that $p$ belongs to a $\sigma$-end $e$ of $T$. Since the configuration $p$ is multitropically generic, Lemma 7 implies that the end $e$ is not marked, i.e., terminates at a point $q \in L_{-\infty}$ different from any point of $\boldsymbol{p}^{\mathrm{b}}$. Consider the configuration $\hat{\boldsymbol{p}}=\left(\hat{\boldsymbol{p}}^{\mathrm{b}}, \hat{\boldsymbol{p}}^{\sharp}\right) \in \Omega\left(\Delta, \alpha+\theta_{k}, \beta-\theta_{k}, g\right)$, where $k=w(e)$ is the weight of $e$, the configuration $\hat{\boldsymbol{p}}^{\mathrm{b}}$ is obtained from $\boldsymbol{p}^{\mathrm{b}}$ by insertion of $q$ in the $k$-th group of points, and $\hat{\boldsymbol{p}}^{\#}=\boldsymbol{p}^{\sharp} \backslash\{p\}$. Since $\boldsymbol{p} \in \mathcal{X}$, the configuration $\hat{\boldsymbol{p}}$ is multi-tropically generic.

Clearly, the curve $T$ belongs to $\mathcal{T}\left(\Delta, \alpha+\theta_{k}, \beta-\theta_{k}, g, \hat{\boldsymbol{p}}\right)$. On the other hand, we can assume that $N$ is chosen so that $\mathcal{T}\left(\Delta, \alpha+\theta_{k}, \beta-\theta_{k}, g, \hat{\boldsymbol{p}}\right) \subset \mathcal{T}(\Delta, \alpha, \beta, g, \boldsymbol{p})$. Thus, the contribution to $\mathrm{W}(\Delta, \alpha, \beta, g)$ of the curves $T \in \mathcal{T}(\Delta, \alpha, \beta, g, \boldsymbol{p})$ such that $p$ belongs to a $\sigma$-end of $T$ is equal to

$$
\sum_{k \geq 1, \beta_{k}>0} \mathrm{~W}\left(\Delta, \alpha+\theta_{k}, \beta-\theta_{k}, g\right) .
$$

Suppose now that $p$ does not belong to any $\sigma$-end of $T$. Since $T$ is nodal, it can be represented in a unique way as a sum of its irreducible subcurves $T^{(1)}, \ldots, T^{(\ell)}$. For any $j=1, \ldots, \ell$, consider a simple parameterization $\left(\Gamma^{(j)}, w^{(j)}, h^{(j)}\right)$ of $T^{(j)}$. Pick a number $c$ such that $a<c<b$. For any curve $\left(\Gamma^{(j)}, w^{(j)}, h^{(j)}\right)$ consider the lift $\Upsilon^{(j)} \subset \Gamma^{(j)}$ of the intersection points of $T^{(j)}$ with the vertical segment $x=c,-\varepsilon \leq$ $y \leq \varepsilon$. Lemma 28 implies that no connected component of $\Gamma^{(j)} \backslash \Upsilon^{(j)}$ has an image intersecting the both halves $R_{a}^{b} \cap\{x<c\}$ and $R_{a}^{b} \cap\{x>c\}$ of $R_{a}^{b}$. If $\Upsilon^{(j)} \neq \varnothing$, then $\Upsilon^{(j)}$ cuts $\Gamma^{(j)}$ in two parts: the image of any connected component in the right part $\Gamma_{\mathcal{R}}^{(j)}$ intersects $R_{a}^{b} \cap\{x>c\}$, and the image of any connected component of the left part $\Gamma_{\mathscr{L}}^{(j)}$ intersects $R_{a}^{b} \cap\{x<c\}$. Any connected component $\Gamma_{\mathscr{L}}^{(j, v)}, v=1, \ldots, \ell_{j}$, gives rise to a parameterized plane tropical curve $\left(\Gamma_{\mathscr{L}}^{(j, v)}, w_{\mathscr{L}}^{(j, \nu)}, h_{\mathscr{L}}^{(j, v)}\right.$ ), where the weight function $w_{\mathscr{L}}^{(j, v)}$ is induced by $w^{(j)}$, and $h_{\mathscr{L}}^{(j, v)}$ is given by a modification of $h^{(j)}$ on the edges cut (without changing the directions of the images of these edges). The image of $\Gamma_{\mathscr{L}}^{(j, v)}$ under $h_{\mathscr{L}}^{(j, v)}$ is obtained from $h^{(j)}\left(\Gamma_{\mathscr{L}}^{(j, v)}\right)$ by the extension of the edges cut by the segment $x=c,-\varepsilon \leq y \leq \varepsilon$ up to horizontal positively directed rays.

For any connected component $\Gamma_{\mathcal{R}}^{(j, \mu)}, \mu=1, \ldots, \ell_{j}^{\prime}$ of $\Gamma_{\mathcal{R}}^{(j)}$, denote by $\widehat{\Gamma}_{\mathcal{R}}^{(j, \mu)}$ the graph obtained from $\Gamma_{\mathcal{R}}^{(j, \mu)}$ by adding a vertex to each cut edge. The graph $\hat{\Gamma}_{\mathcal{R}}^{(j, \mu)}$ gives 
rise to a parameterized plane tropical curve $\left(\widehat{\Gamma}_{\mathcal{R}}^{(j, \mu)}, w_{\mathcal{R}}^{(j, \mu)}, h_{\mathcal{R}}^{(j, \mu)}\right)$, where again the weight function $w_{\mathcal{R}}^{(j, \mu)}$ is induced by $w^{(j)}$, the restriction of $h_{\mathcal{R}}^{(j, \mu)}$ on $\Gamma_{\mathcal{R}}^{(j, \mu)}$ is given by a modification of $h^{(j)}$ on the edges cut (without changing the directions of the images of these edges), and the image under $h_{\mathcal{R}}^{(j, \mu)}$ of any added vertex belongs to $L_{-\infty}$. The image of $\widehat{\Gamma}_{\mathcal{R}}^{(j, \mu)}$ under $h_{\mathcal{R}}^{(j, \mu)}$ is obtained from $h^{(j)}\left(\Gamma_{\mathcal{R}}^{(j, \mu)}\right)$ by the extension of the edges cut by the segment $x=c,-\varepsilon \leq y \leq \varepsilon$ up to horizontal negatively directed rays. Denote by $T^{\prime}$ the plane tropical curve defined by the collection of all parameterized plane tropical curves $\left(\hat{\Gamma}_{\mathcal{R}}^{(j, \mu)}, w_{\mathcal{R}}^{(j, \mu)}, h_{\mathcal{R}}^{(j, \mu)}\right), j=1, \ldots, \ell, \mu=1, \ldots, \ell_{j}^{\prime}$. We say that $T^{\prime}$ is the derivation of $T$.

In the case that $\Upsilon^{(j)}$ is empty, then $h^{(j)}\left(\Gamma^{(j)}\right) \subset\{x<c\}$, and we use the notation $\left(\Gamma_{\mathscr{L}}^{(j, 1)}, w_{\mathscr{L}}^{(j, 1)}, h_{\mathscr{L}}^{(j, 1)}\right)$ for the parameterized plane tropical curve $\left(\Gamma^{(j)}, w^{(j)}, h^{(j)}\right)$.

Since the half-plane $x<c$ contains only one point of $\boldsymbol{p}^{\sharp}$, Lemma 23 implies that

- all the curves $\left(\Gamma_{\mathscr{L}}^{(j, v)}, w_{\mathscr{L}}^{(j, v)}, h_{\mathscr{L}}^{(j, v)}\right), j=1, \ldots, \ell, v=1, \ldots, \ell_{j}$, are of genus 0 ,

- among the curves $\left(\Gamma_{\mathscr{L}}^{(j, v)}, w_{\mathscr{L}}^{(j, v)}, h_{\mathscr{L}}^{(j, v)}\right), j=1, \ldots, \ell, v=1, \ldots, \ell_{j}$, there exists a curve $(\Gamma, w, h)$ such that $\Gamma$ has exactly one end whose image under $h$ points upwards (i.e., has the direction either $(0,1)$ or $(1,1))$, and exactly one end whose image points downwards (i.e., has the direction either $(0,-1)$ or $(-1,-1))$,

- the image under $h$ of any left end of $\Gamma$ terminates at a point of $\boldsymbol{p}^{\mathrm{b}}$,

- for any curve $\left(\Gamma_{\mathscr{L}}^{(j, v)}, w_{\mathscr{L}}^{(j, v)}, h_{\mathscr{L}}^{(j, v)}\right)$ which is different from $(\Gamma, w, h)$ the image $h_{\mathscr{L}}^{(j, v)}\left(\Gamma_{\mathscr{L}}^{(j, v)}\right)$ is a horizontal straight line; such curves $\left(\Gamma_{\mathscr{L}}^{(j, v)}, w_{\mathscr{L}}^{(j, v)}, h_{\mathscr{L}}^{(j, v)}\right)$ are called horizontal.

In particular, the Newton polygon $\Delta^{\prime}$ of $T^{\prime}$ is the T-peeling $l_{T}(\Delta)$ of $\Delta$ for certain $\neg \subset\{\overrightarrow{0}, \overrightarrow{-1}\}$. Denote by $\sigma^{\prime}$ the vertical left-most side of $\Delta^{\prime}$.

Let $\left(\boldsymbol{p}^{\prime}\right)^{\mathrm{b}} \subset \boldsymbol{p}^{\mathrm{b}}$ be the subconfiguration formed by the images of marked terminal points of horizontal curves, and let $\alpha^{\prime} \leq \alpha$ be the corresponding sequence in $\mathcal{C}$. Since $T$ is nodal, the images of terminal points of left ends of graphs $\Gamma_{\mathcal{R}}^{(j)}$ are disjoint from the images of terminal points of left ends of $\Gamma$. Among the left ends of graphs $\Gamma_{\mathcal{R}}^{(j)}$ consider the edges whose images terminate at points different from the points of $\boldsymbol{p}^{\mathrm{b}}$, and denote by $\beta^{\prime}$ the sequence determined by the weights of the edges considered. Since the image under $h$ of any left end of $\Gamma$ terminates at a point of $\boldsymbol{p}^{\mathrm{b}}$, we have $\beta^{\prime} \geq \beta$. Furthermore, $J \alpha^{\prime}+J \beta^{\prime}=\left|\sigma^{\prime}\right|$. Counting the edges cut by the segment $x=c,-\varepsilon \leq y \leq \varepsilon$, we obtain that the genus $g^{\prime}$ of $T^{\prime}$ is equal to $g-\left\|\beta^{\prime}-\beta\right\|+1$. The curve $T^{\prime}$ belongs to $\mathcal{T}\left(\Delta^{\prime}, \alpha^{\prime}, \beta^{\prime}, g^{\prime}, \boldsymbol{p}^{\prime}\right)$, where $\boldsymbol{p}^{\prime}=\left(\left(\boldsymbol{p}^{\prime}\right)^{\mathrm{b}},\left(\boldsymbol{p}^{\prime}\right)^{\sharp}\right)$, and $\left(\boldsymbol{p}^{\prime}\right)^{\sharp}$ is obtained from $p^{\sharp}$ by removing the point $p$, and all the edges of $T^{\prime}$ are of odd weights.

Describe now the inverse procedure. Fix two elements $\alpha^{\prime}, \beta^{\prime} \in \mathcal{C}$ such that $\alpha^{\prime} \leq \alpha, \beta^{\prime} \geq \beta$, and $J \alpha^{\prime}+J \beta^{\prime}=\left|\sigma^{\prime}\right|$. Put $g^{\prime}=g-\left\|\beta^{\prime}-\beta\right\|+1$. Choose 

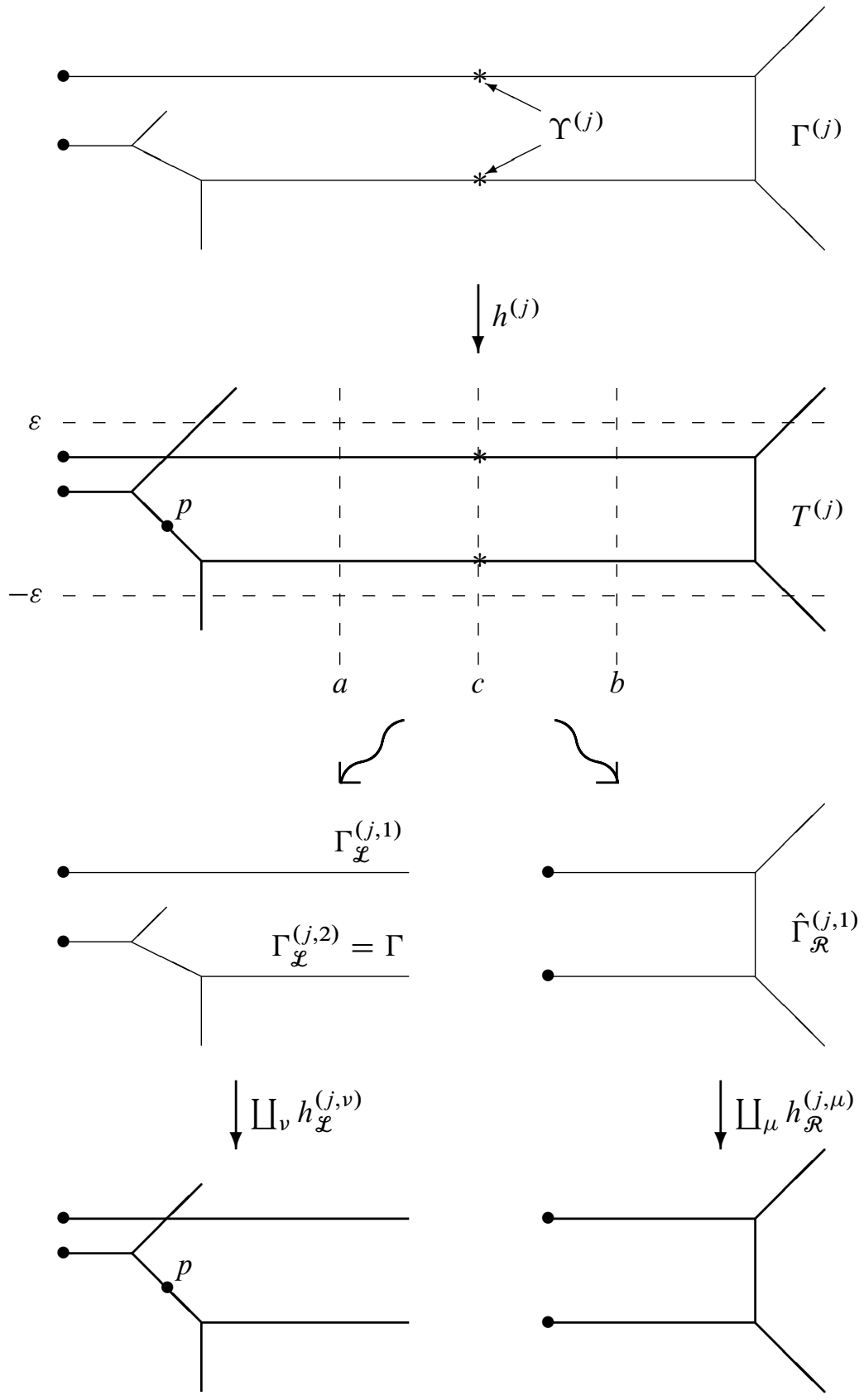

Figure 6. Cut in the proof of Theorem 3. 
a subconfiguration $\left(\boldsymbol{p}^{\prime}\right)^{\mathrm{b}} \subset \boldsymbol{p}^{\mathrm{b}}$ such that $\left(\boldsymbol{p}^{\prime}\right)^{\mathrm{b}}$ corresponds to the sequence $\alpha^{\prime}$ (the number of such subconfigurations $\left(\boldsymbol{p}^{\prime}\right)^{b}$ is $\left.\left(\begin{array}{c}\alpha \\ \alpha^{\prime}\end{array}\right)\right)$. Consider the configuration $\boldsymbol{p}^{\prime}=$ $\left(\boldsymbol{p}^{\prime}\right)^{\mathrm{b}} \cup\left(\boldsymbol{p}^{\prime}\right)^{\sharp}$, where $\left(\boldsymbol{p}^{\prime}\right)^{\sharp}$, as before, is obtained from $\boldsymbol{p}^{\sharp}$ by removing the point $p$. By Lemma 31, the configuration $\boldsymbol{p}^{\prime}$ is multi-tropically generic. Furthermore, by Lemma 26 the set $\mathcal{T}\left(\Delta^{\prime}, \alpha^{\prime}, \beta^{\prime}, g^{\prime}, \boldsymbol{p}^{\prime}\right)$ is finite, and we can assume that the first coordinate of $p$ is much less than the first coordinates of the vertices of all the curves in $\mathcal{T}\left(\Delta^{\prime}, \alpha^{\prime}, \beta^{\prime}, g^{\prime}, p^{\prime}\right)$.

Pick a curve $T^{\prime} \in \mathcal{T}\left(\Delta^{\prime}, \alpha^{\prime}, \beta^{\prime}, g^{\prime}, \boldsymbol{p}^{\prime}\right)$ without edges of even weight. Let $\left(\hat{\Gamma}_{\mathcal{R}}^{(i)}, w_{\mathcal{R}}^{(i)}, h_{\mathcal{R}}^{(i)}\right), i=1, \ldots, m$, be simple parameterizations of irreducible subcurves of $T^{\prime}$. Among the non-marked left ends of $\coprod_{i=1}^{m} \widehat{\Gamma}_{\mathcal{R}}^{(i)}$ choose $\left\|\beta^{\prime}-\beta\right\|$ ends whose weights fit the sequence $\beta^{\prime}-\beta$ (the number of such choices is $\left(\begin{array}{c}\beta^{\prime} \\ \beta\end{array}\right)$ ), and denote the chosen set by $\mathcal{E}$. Lemma 29 provides a parameterized plane tropical curve $(\Gamma, w, h)$ such that

- $(\Gamma, w, h)$ is of genus 0 and has exactly two ends whose images under $h$ are not horizontal; these ends are of weight 1 , and their images point in the directions of outward normal vectors of the sides $T(\Delta, T)$ and $\perp(\Delta, T)$ of $\Delta$ (see the proof of Lemma 31 for notation),

- the images of left ends of $\Gamma$ terminate at the points of $\boldsymbol{p}^{b} \backslash\left(\boldsymbol{p}^{\prime}\right)^{b}$, and the weights of these left ends are given by the sequence $\alpha-\alpha^{\prime}$,

- the ends of $\Gamma$ whose images are horizontal positively directed fit the ends belonging to $\&$ and have the corresponding weights,

- $p \in h(\Gamma)$.

In the same way as in the proof of Lemma 31 , we can glue $(\Gamma, w, h)$ with the curves $\left(\hat{\Gamma}_{\mathcal{R}}^{(i)}, w_{\mathcal{R}}^{(i)}, h_{\mathcal{R}}^{(i)}\right), i=1, \ldots, m$, and obtain a collection of parameterizations of plane tropical curves $T^{(1)}, \ldots, T^{(\ell)}$ whose sum $T$ belongs to $\mathcal{T}(\Delta, \alpha, \beta, g, \boldsymbol{p})$. Since $\boldsymbol{p}$ is multi-tropically generic, Lemma 29 implies that the curve $(\Gamma, w, h)$ is defined by the above properties uniquely. Furthermore, the initial curve $T^{\prime}$ is the derivation of $T$. The multiplicative contribution of the trivalent vertices of $\Gamma$ to the Welschinger multiplicity of $T$ is 1 , and we finally conclude that, for given $\alpha^{\prime}$ and $\beta^{\prime}$, the contribution to $\mathrm{W}(\Delta, \alpha, \beta, g)$ of the curves $T \in \mathcal{T}(\Delta, \alpha, \beta, g, \boldsymbol{p})$ such that $p$ does not belong to any $\sigma$-end of $T$ is equal to $\left(\begin{array}{c}\alpha \\ \alpha^{\prime}\end{array}\right)\left(\begin{array}{c}\beta^{\prime} \\ \beta\end{array}\right) \mathrm{W}\left(\Delta^{\prime}, \alpha^{\prime}, \beta^{\prime}, g^{\prime}\right)$.

5.3. Proof of Theorem 4. The proof goes in the same way as for Theorem 3 . The only modification concerns the second sum in the right-hand side of (4): assuming that a plane tropical curve $T^{\prime} \in \mathcal{T}\left(\Delta^{\prime}, \alpha^{\prime}, \beta^{\prime}, g^{\prime}, \boldsymbol{p}^{\prime}\right)$ is the derivation of $T \in \mathcal{T}^{\text {irr }}(\Delta, \alpha, \beta, g, \boldsymbol{p})$, we have to describe possible irreducible subcurves of $T^{\prime}$ and their contribution to the formula. The first (respectively, second) coefficient in 
the second sum of the formula reflects the distribution of the points of $\left(\boldsymbol{p}^{\prime}\right)^{\mathrm{b}}$ (respectively, $\left.\left(\boldsymbol{p}^{\prime}\right)^{\#}\right)$ among the irreducible subcurves of $T^{\prime}$. The conditions on the numbers $\alpha^{\prime}, \beta^{\prime}, g^{\prime}$ and $\alpha^{(i)}, \beta^{(i)}, g^{(i)}, i=1, \ldots, m$, come from the conditions in Theorem 3, and the inequalities $\left\|\tilde{\beta}^{(i)}\right\|>0$ mean that, for each irreducible subcurve $T^{(i)}$, its simple parameterization $\left(\widehat{\Gamma}_{\mathcal{R}}^{(i)}, w_{\mathcal{R}}^{(i)}, h_{\mathcal{R}}^{(i)}\right)$ must glue with the curve $(\Gamma, w, h)$ (in the notation of the proof of Theorem 3 ).

\section{Concluding remarks}

6.1. Generating functions. Let $\Delta$ be one of the polygons shown in Figure 1, and $\Sigma$ the real toric Del Pezzo surface defined by $\Delta$. We say that a convex lattice polygon $\Delta^{0}$ has the same shape as $\Delta$, if $\Delta$ and $\Delta^{0}$ have the same number of sides, and any side of $\Delta^{0}$ is parallel to a side of $\Delta$. Let $\Xi_{\Sigma}$ be the set which consists of all convex lattice polygons (considered up to parallel translation) having the same shape as $\Delta$ and their 7 -peelings ( $\neg \subset\{\overrightarrow{0}, \overrightarrow{-1}\}$ being $\Delta$-admissible). The set $\Xi_{\Sigma}$ is a commutative semigroup with respect to the Minkowsky sum.

Following [4], [9] introduce two generating functions

$$
\begin{aligned}
Z_{\Sigma}(w, x, y, z) & =\sum_{\substack{g \in \mathbb{Z}, \Delta \in \Xi_{\Sigma} \Sigma \\
\alpha, \beta \in \mathcal{C}, J \alpha+J \beta=|\sigma(\Delta)|}} \mathrm{W}(\Delta, \alpha, \beta, g) v^{\Delta} w^{g-1} \frac{x^{\alpha}}{\alpha !} y^{\beta} \frac{z^{r}}{r !}, \\
Z_{\Sigma}^{\mathrm{irr}}(w, x, y, z) & \sum_{\substack{g \geq 0, \Delta \in \Xi_{\Sigma} \\
\alpha, \beta \in \mathcal{C}, J \alpha+J \beta=|\sigma(\Delta)|}} \mathrm{W}^{\mathrm{irr}}(\Delta, \alpha, \beta, g) v^{\Delta} w^{g-1} \frac{x^{\alpha}}{\alpha !} y^{\beta} \frac{z^{r}}{r !},
\end{aligned}
$$

where $\sigma(\Delta)$ is the intersection of $\Delta$ with its left vertical supporting line, $x$ and $y$ are infinite sequences of variables, and $r$ is defined by (1). These generating functions can be seen as formal series in variables $w, z$ and multi-variables $x, y$ with coefficients in the Novikov ring of the semigroup $\Xi_{\Sigma}$. Using the same arguments as in [9], Section 6.4, and [4], Section 5.3, one can check that these generating functions are related by the identity $Z_{\Sigma}=\exp Z_{\Sigma}^{\text {irr }}$ and satisfy the differential equations

$$
\begin{aligned}
& \sum_{\top \subset\{\overrightarrow{0}, \overrightarrow{-1}\}} v^{l \top}\left(\frac{\partial}{\partial z}-\sum_{k=1}^{\infty} y_{k} \frac{\partial}{\partial x_{k}}\right) Z_{\Sigma}=\frac{1}{w} \cdot \operatorname{res}_{t=0} \exp \sum_{k=1}^{\infty}\left(t^{-k} x_{k}+w t^{k} \frac{\partial}{\partial y_{k}}\right) Z_{\Sigma}, \\
& \left.\sum_{ד \subset\{\overrightarrow{0}, \overrightarrow{-1}\}} v^{l}\right\urcorner\left(\frac{\partial}{\partial z}-\sum_{k=1}^{\infty} y_{k} \frac{\partial}{\partial x_{k}}\right) Z_{\Sigma}^{\mathrm{irr}} \\
& =\frac{1}{w} \cdot \operatorname{res}_{t=0} \exp \left(\sum_{k=1}^{\infty}\left(t^{-k} x_{k}+\left.Z_{\Sigma}^{\mathrm{irr}}\right|_{y_{k} \mapsto y_{k}+w t^{k}}\right)-Z_{\Sigma}^{\mathrm{irr}}\right),
\end{aligned}
$$


where $\left.Z_{\Sigma}^{\mathrm{irr}}\right|_{y_{k} \mapsto y_{k}+w t^{k}}$ stands for $Z_{\Sigma}^{\mathrm{irr}}$ with $y_{k}$ replaced by $y_{k}+w t^{k}$, and

$$
v^{l_{\top}} v^{\Delta}= \begin{cases}v^{l_{\top}(\Delta)}, & \text { if } ד \text { is } \Delta-\text { admissible, } \\ 0, & \text { otherwise. }\end{cases}
$$

6.2. Non-invariance in the classical setting. The absence of invariants of topological nature as mentioned in Introduction can be illustrated by the following examples.

Real irreducible plane curves of degree $d$ and genus $g$ passing through a generic configuration of $3 d+g-1$ real points form a finite set. If $g>0$, then under variation of the point configuration, this set is subject to codimension one events in which a pair of real curves with the same embedded topology disappears turning into a pair of imaginary conjugate curves, or vice versa. In the case of elliptic quartics ( $d=4$, $g=1$ ), it is shown in [5], Theorem 3.1. Gluing elliptic quartics with one or several lines, it is not difficult to construct examples of higher degree and genus. The example with elliptic quartics shows also that the number $\mathrm{W}\left(\Delta\left(5 \mathbb{P}^{1}\right),(0),(5), 0\right)$ does not lift up to the classical setting as an invariant formulated in purely topological terms.

Another example, demonstrating the same phenomenon, is as follows. Consider plane rational curves of degree $d$ which pass through $3 d+1-b_{1}-b_{2}$ generic points outside a line $\mathbb{P}^{1} \subset \mathbb{P}^{2}$ and have two non-fixed tangency points with $\mathbb{P}^{1}$, one of intersection order $b_{1}$ and the other of intersection order $b_{2}$, where $b_{1}$ and $b_{2}$ are distinct, odd, and satisfy the inequality $b_{1}+b_{2}<d$. Under variation of the point configuration, the collision of the two tangency points into one tangency point of order $b_{1}+b_{2}$ is an event of codimension one. Crossing such a wall leads to appearance, or disappearance, of two real curves which have the same embedded topology (even with respect to $\mathbb{P}^{1}$ ).

One more phenomenon is the change of the Welschinger multiplicity of precisely one member of the set of curves. Consider real plane rational curves of degree $d \geq 5$ passing through $a \geq 3$ fixed generic real points on $\mathbb{P}^{1} \subset \mathbb{P}^{2}$ and $3 d-1-a$ generic real points in $\mathbb{P}^{2} \backslash \mathbb{P}^{1}$. Here we observe the following codimension one event: precisely one of the curves splits into $D+\mathbb{P}^{1}$, where $D$ is tangent to $\mathbb{P}^{1}$ at one point and transversal to it at $d-3$ other points. On one side of such a wall the tangency point turns into a solitary node, and on the other side into a crossing point, whereas the other singularities do not change.

The above arguments do not exclude the existence of relative real algebraic enumerative invariants in other situations. For example, such invariants were introduced by Welschinger [12] in the case of one simple tangency constraint with respect to a smooth null-homologous curve. 


\section{References}

[1] L. Caporaso and J. Harris, Counting plane curves of any genus. Invent. Math. 131 (2) (1998), 345-392. Zbl 0934.14040 MR 1608583

[2] A. Gathmann and H. Markwig, The numbers of tropical plane curves through points in general position. J. Reine Angew. Math. 602 (2007), 155-177. Zbl 1115.14049 MR 2300455

[3] A. Gathmann and H. Markwig, The Caporaso-Harris formula and plane relative Gromov-Witten invariants in tropical geometry. Math. Ann. 338 (4) (2007), 845-868. Zbl 1128.14040 MR 2317753

[4] E. Getzler, Intersection theory on $\overline{\mathcal{M}}_{1,4}$ and elliptic Gromov-Witten invariants. J. Amer. Math. Soc. 10 (4) (1997), 973-998. Zbl 0909.14002 MR 1451505

[5] I. Itenberg, V. Kharlamov and E. Shustin, Welschinger invariant and enumeration of real rational curves. Internat. Math. Res. Notices 49 (2003), 2639-2653. Zbl 1083.14523 MR 2012521

[6] G. Mikhalkin, Counting curves via the lattice paths in polygons. C. R. Acad. Sci. Paris Sér. I 336 (8) (2003), 629-634. Zbl 1027.14026 MR 1988122

[7] G. Mikhalkin, Enumerative tropical algebraic geometry in $\mathbb{R}^{2}$. J. Amer. Math. Soc. 18 (2005), 313-377. Zbl 1092.14068 MR 2137980

[8] E. Shustin, A tropical approach to enumerative geometry. Algebra i Analiz 17 (2) (2005), 170-214. Zbl 1100.14046 MR 2159589

[9] R. Vakil, Counting curves on rational surfaces. Manuscripta Math. 102 (1) (2000), 53-84. Zbl 0967.14036 MR 1771228

[10] J.-Y. Welschinger, Invariants of real rational symplectic 4-manifolds and lower bounds in real enumerative geometry. C. R. Acad. Sci. Paris Sér. I 336 (2003), 341-344. Zbl 1042.57018 MR 1976315

[11] J.-Y. Welschinger, Invariants of real symplectic 4-manifolds and lower bounds in real enumerative geometry. Invent. Math. 162 (1) (2005), 195-234. Zbl 1082.14052 MR 2198329

[12] J.-Y. Welschinger, Towards relative invariants of real symplectic four-manifolds. Geom. Funct. Anal. 16 (5) (2006), 1157-1182. Zbl 1107.53059 MR 2276536

Received February 15, 2007

Ilia Itenberg, Université Louis Pasteur et IRMA, 7, rue René Descartes, 67084 Strasbourg Cedex, France

E-mail: itenberg@math.u-strasbg.fr

Viatcheslav Kharlamov, Université Louis Pasteur et IRMA, 7, rue René Descartes, 67084 Strasbourg Cedex, France

E-mail: kharlam@math.u-strasbg.fr

Eugenii Shustin, School of Mathematical Sciences, Raymond and Beverly Sackler Faculty of Exact Sciences, Tel Aviv University, Ramat Aviv, 69978 Tel Aviv, Israel

E-mail: shustin@post.tau.ac.il 